\title{
Escape rates and physically relevant measures for billiards with small holes
}

Mark Demers

Fairfield University, mdemers@fairfield.edu

Paul Wright

Lai-Sang Young

Follow this and additional works at: https://digitalcommons.fairfield.edu/mathandcomputersciencefacultypubs

Copyright 2010 Springer-Verlag

\section{Peer Reviewed}

\section{Repository Citation}

Demers, Mark; Wright, Paul; and Young, Lai-Sang, "Escape rates and physically relevant measures for billiards with small holes" (2010). Mathematics Faculty Publications. 43.

https://digitalcommons.fairfield.edu/mathandcomputerscience-facultypubs/43

\section{Published Citation}

Mark Demers, Paul Wright and Lai-Sang Young, "Escape rates and physically relevant measures for billiards with small holes," Communications in Mathematical Physics 294: 2 (2010), 353-388.

This Article is brought to you for free and open access by the Mathematics Department at DigitalCommons@Fairfield. It has been accepted for inclusion in Mathematics Faculty Publications by an authorized administrator of DigitalCommons@Fairfield. For more information, please contact digitalcommons@fairfield.edu. 


\section{Escape Rates and Physically Relevant Measures for Billiards with Small Holes}

\author{
Mark Demers* $\quad$ Paul Wright ${ }^{\dagger} \quad$ Lai-Sang Young ${ }^{\ddagger}$
}

January 21, 2010

This paper is about leaky dynamical systems, or dynamical systems with holes. Consider a dynamical system defined by a map or a flow on a phase space $M$, and let $H \subset M$ be a hole through which orbits escape, that is to say, once an orbit enters $H$, we stop considering it from that point on. Starting from an initial probability distribution $\mu_{0}$ on $M$, mass will leak out of the system as it evolves. Let $\mu_{n}$ denote the distribution remaining at time $n$. The most basic question one can ask about a leaky system is its rate of escape, i.e. whether $\mu_{n}(M) \sim \vartheta^{n}$ for some $\vartheta$. Another important question concerns the nature of the remaining distribution. One way to formulate that is to normalize $\mu_{n}$, and to inquire about properties of $\mu_{n} / \mu_{n}(M)$ as $n$ tends to infinity. Such limiting distributions, when they exist, are not invariant; they are conditionally invariant, meaning they are invariant up to a normalization. Comparisons of systems with small holes with the corresponding closed systems, i.e. systems for which the holes have been plugged, are also natural. These are some of the questions we will address in this paper.

*Department of Mathematics and Computer Science, Fairfield University. Email: mdemers@fairfield.edu. This research is partially supported by NSF grant DMS-0801139.

$\dagger$ Department of Mathematics, University of Maryland. Email: paulrite@math.umd.edu. This research is partially supported by an NSF Mathematical Sciences Postdoctoral Research Fellowship. This author would also like to thank The Courant Institute of Mathematical Sciences, New York University, where he was affiliated when this project began.

${ }^{\ddagger}$ Courant Institute of Mathematical Sciences, New York University. Email: lsy@cims.nyu.edu. This research is partially supported by a grant from the NSF.

The authors would like to thank MSRI, Berkeley, and ESI, Vienna, where part of this work was carried out. 
We do not consider these questions in the abstract, however; for a review paper in this direction, see $[\mathrm{DY}]$. Our context here is that of billiard systems with small holes. Specifically, we carry out our analysis for the collision map of a 2-dimensional periodic Lorentz gas, and expect our results to be extendable to other dispersing billiards. Our holes are "physical" holes, in the sense that they are derived from holes in the physical domain of the system, i.e., the billiard table: we consider both convex holes away from the scatterers and holes that live on the boundaries of the scatterers. The holes considered in this paper are very small, but their placements are immaterial. For these leaky systems, we prove that there is a common rate of escape and a common limiting distribution for a large class of natural initial distributions including those with densities with respect to Liouville measure. These conditionally invariant measures, therefore, can be viewed as characteristic of the leaky systems in question, in a way that is analogous to physical measures or SRB measures for closed systems. We show, in fact, that as hole size tends to zero, these measures tend to the natural invariant measure of the corresponding closed billiard system.

Our proof involves constructing a Markov tower extension with a special property over the billiard map, the new requirement being that it respects the hole. Let us backtrack a little for readers not already familiar with these ideas: In much the same way that Markov partitions have proved to be very useful in the study of Anosov and Axiom A diffeomorphisms, it was shown, beginning with $[\mathrm{Y}]$ and continued in a number of other papers, that many systems with sufficiently strong hyperbolic properties (but which are not necessarily uniformly hyperbolic) admit countable Markov extensions. Roughly speaking, these extensions behave like countable state Markov chains "with nonlinearity"; they have considerably simpler structures than the original dynamical system. The idea behind this work is that escape dynamics are much simpler in a Markov setting when the hole corresponds to a collection of "states"; this is what we mean by the Markov extension "respecting the hole." All this is not for free, however. We pay a price with a somewhat elaborate construction of the tower, and again when we pass the information back to the billiard system, in exchange for having a Markov structure to work with in the treatment of the hole.

There are advantages to this route of proof: First, once a Markov extension is constructed for a system, it can be used many times over for entirely different purposes. For the billiard maps studied here, these extensions were constructed in $[Y]$; our main task is to adapt them to holes. Second, once results on escape dynamics are established on towers, they apply to all Markov extensions. Here, the desired results are already known in a special case, namely expanding towers $[\mathrm{BDM}]$; we need to extend them to the general, hyperbolic setting. What we propose here is a unified, generic approach for dealing with holes in dynamical systems, one that can, in principle, be carried out for all systems that admit Markov towers. Such systems include logistic maps, rank one attractors including the Hénon family, piecewise hyperbolic maps and other dispersing billiards in 2 or more dimensions.

Conditionally invariant measures were first introduced in probabilistic settings, namely countable state Markov chains and topological Markov chains, beginning with [V] and more recently in [FKMP] and [CMS3]. In this setting, such measures are called quasi-stationary distributions and the existence of a Yaglom limit corresponds to the limit $\mu_{n} / \mu_{n}(M)$, which we use here to identify a physical conditionally invariant measure for the leaky system.

The first works to study deterministic systems with holes took advantage of finite Markov partitions. These include: Expanding maps on $\mathbb{R}^{n}$ with holes which are elements of a finite 
Markov partition [PY, CMS1, CMS2]; Smale horseshoes [C1, C2]; Anosov diffeomorphisms [CM1, CM2, CMT1, CMT2]; billiards with convex scatterers satisfying a non-eclipsing condition $[\mathrm{LM}, \mathrm{R}]$ and large parameter logistic maps whose critical point maps out of the interval $[\mathrm{HY}]$. In the latter two, the holes are chosen in such a way that the surviving dynamics are uniformly expanding or hyperbolic with Markov partitions. First results which drop Markov requirements on the map include piecewise expanding maps of the interval [BaK, CV, LiM, D1, BDM]; Misiurewicz [D2] and Collet-Eckmann [BDM] maps with generic holes; and piecewise uniformly hyperbolic maps [DL]. The tower construction is used in the one-dimensional studies $[\mathrm{D} 1, \mathrm{D} 2, \mathrm{BDM}]$. Typically a restriction on the size of the hole is introduced in order to control the dynamics when a finite Markov partition is absent.

General conditions ensuring the existence of conditionally invariant measures are first given in $[\mathrm{CMM}]$. The physical relevance of such measures, however, is unclear without further qualifications. As noted in [DY], under very weak assumptions on the dynamical system, many such measures exist: for any prescribed rate of escape, one can construct infinitely many conditionally invariant densities. This is the reason for the emphasis placed in this paper on the limit $\mu_{n} / \mu_{n}(M)$, which identifies a unique, physically relevant conditionally invariant measure.

This paper is organized as follows: Our results are formulated in Sect. 1. In Sects. 2 and 3 , the geometry of billiard maps and holes are looked at carefully as we modify previous constructions to give a generalized horseshoe that respects the hole. Out of this horseshoe, a Markov tower extension is constructed and results on escape dynamics on it proved; this is carried out in Sects. 4 and 5. These results are passed back to the billiard system in Sect. 6, where the remaining theorems are also proved.

\section{Formulation of Results}

\section{$1.1 \quad$ Basic definitions}

We consider a closed dynamical system defined by a self-map $f$ of a manifold $M$, and let $H \subset M$ be a hole through which orbits escape, i.e., we stop considering an orbit once it enters $H$. In this paper we are primarily concerned with holes that are open subsets of the phase space; they are not too large and generally not $f$-invariant. We will refer to the triplet $(f, M, H)$ as a leaky system.

First we introduce some notation. Let $\stackrel{\circ}{M}=M \backslash H$. At least to begin with, let us make

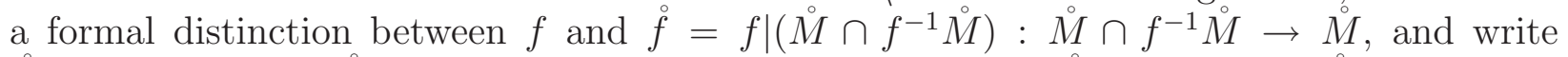
$\stackrel{\circ}{f}^{n}=f^{n} \mid\left(\bigcap_{i=0}^{n} f^{-i} \stackrel{\circ}{M}\right)$. Let $\eta$ be a probability measure on $\stackrel{\circ}{M}$. We define $\stackrel{\circ}{f}_{*} \eta$ to be the

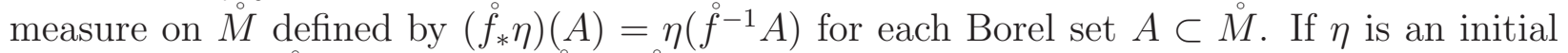
distribution on $\stackrel{\circ}{M}$, then $\eta^{(n)}:=f_{*}^{n} \eta /\left|f_{*}^{n} \eta\right|$ is the normalized distribution of points remaining in $\stackrel{\circ}{M}$ after $n$ units of time.

Given an initial distribution $\eta$, the most basic question is the rate at which mass is leaked out of the system. We define the escape rate starting from $\eta$ to be $-\log \vartheta(\eta)$ where

$$
\log \vartheta(\eta)=\lim _{n \rightarrow \infty} \frac{1}{n} \log \eta\left(\bigcap_{i=0}^{n} f^{-i} \stackrel{\circ}{M}\right) \quad \text { assuming such a limit exists. }
$$


Another basic object is the limiting distribution $\eta^{(\infty)}$ defined to be $\eta^{(\infty)}=\lim _{n \rightarrow \infty} \eta^{(n)}$ if this weak limit exists. Of particular interest is when there is a number $\vartheta_{*}$ and a probability measure $\mu_{*}$ with the property that for all $\eta$ in a large class of natural initial distributions (such as those having densities with respect to Lebesgue measure), we have $\vartheta(\eta)=\vartheta_{*}$ and $\eta^{(\infty)}=\mu_{*}$. In such a situation, $\mu_{*}$ can be thought of as a physical measure for the leaky system $(f, M, H)$, in analogy with the idea of physical measures for closed systems.

A Borel probability measure $\eta$ on $M$ is said to be conditionally invariant if it satisfies $\stackrel{\circ}{*}_{*} \eta=\vartheta \eta$ for some $\vartheta \in(0,1]$. Clearly, the escape rate of a conditionally invariant measure $\eta$ is well defined and is equal to $-\log \vartheta$. Most leaky dynamical systems admit many conditionally invariant measures; see [DY]. In particular, limiting distributions, when they exist, are often conditionally invariant; they are among the more important conditionally invariant measures from an observational point of view.

Finally, when a physical measure $\eta$ for a leaky system $(f, M, H)$ has absolutely continuous conditional measures on the unstable manifolds of the underlying closed system $(f, M)$, we will call it an SRB measure for the leaky system, in analogy with the idea of SRB measures for closed systems.

\subsection{Setting of present work}

The underlying closed dynamical system here is the billiard map associated with a 2dimensional periodic Lorentz gas. Let $\left\{\Gamma_{i}: i=1, \cdots, d\right\}$ be pairwise disjoint $C^{3}$ simplyconnected curves on $\mathbb{T}^{2}$ with strictly positive curvature, and consider the billiard flow on the "table" $X=\mathbb{T}^{2} \backslash \bigcup_{i}\left\{\right.$ interior $\left.\Gamma_{i}\right\}$. We assume the "finite horizon" condition, which imposes an upper bound on the number of consecutive tangential collisions with $\cup \Gamma_{i}$. The phase space of the unit-speed billiard flow is $\mathcal{M}=\left(X \times \mathbb{S}^{1}\right) / \sim$ with suitable identifications at the boundary. Let $M=\cup_{i} \Gamma_{i} \times\left[-\frac{\pi}{2}, \frac{\pi}{2}\right] \subset \mathcal{M}$ be the cross-section to the billiard flow corresponding to collision with the scatterers, and let $f: M \rightarrow M$ be the Poincaré map. The coordinates on $M$ are denoted by $(r, \varphi)$ where $r \in \cup \Gamma_{i}$ is parametrized by arc length and $\varphi$ is the angle a unit tangent vector at $r$ makes with the normal pointing into the domain $X$. We denote by $\nu$ the invariant probability measure induced on $M$ by Liouville measure on $\mathcal{M}$, i.e., $d \nu=c \cos \varphi d r d \varphi$ where $c$ is the normalizing constant.

We consider the following two types of holes:

Holes of Type $I$. In the table $X$, a hole $\sigma$ of this type is an open interval in the boundary of a scatterer. When $q_{0} \in \cup \Gamma_{i}$, we refer to $\left\{q_{0}\right\}$ as an infinitesimal hole, and let $\Sigma_{h}\left(q_{0}\right)$ denote the collection of all open intervals $\sigma \subset \cup \Gamma_{i}$ in the $h$-neighborhood of $q_{0}$. A hole $\sigma$ in $X$ of this type corresponds to a set $H_{\sigma} \subset M$ of the form $(a, b) \times\left[-\frac{\pi}{2}, \frac{\pi}{2}\right]$.

Holes of Type II. A hole $\sigma$ of this type is an open convex subset of $X$ away from $\cup_{i} \Gamma_{i}$ and bounded by a $C^{3}$ simple closed curve with strictly positive curvature. As above, we regard $\left\{q_{0}\right\} \subset X \backslash \cup \Gamma_{i}$ as an infinitesimal hole, and use $\Sigma_{h}\left(q_{0}\right)$ to denote the set of all $\sigma$ in the $h$-neighborhood of $q_{0}$. In this case, $\sigma \subset X$ does not correspond directly to a set in $M$. Rather, $\sigma$ corresponds directly to a set in $\mathcal{M}$, the phase space for the billiard flow, and we must make a choice as to which set in the cross section $\mathrm{M}$ will represent the hole for the billiard map. There is a well defined set $B_{\sigma} \subset M$ consisting of all $(r, \varphi)$ whose trajectories under the billiard flow on $\mathcal{M}$ will enter $\sigma \times \mathbb{S}^{1}$ before reaching $M$ again. Thus $H_{\sigma}=f\left(B_{\sigma}\right)$ 
is a natural candidate for the hole in $M$ representing $\sigma$, and will be taken as such in this work. However, it would also have been possible to take $B_{\sigma}$ as the representative set. The geometry of $B_{\sigma}$ and $H_{\sigma}$ in phase space will be discussed in detail in Sect. 3.1. Also, we note that the requirement that $\partial \sigma$ be a $C^{3}$ simple closed curve with strictly positive curvature can be considerably relaxed. It is even possible to allow some holes $\sigma$ that are not convex. See the remark at the end of Sect. 3.1.

\subsection{Statement of results}

Let $\mathcal{G}=\mathcal{G}\left(H_{\sigma}\right)$ denote the set of finite Borel measures $\eta$ on $M$ that are absolutely continuous with respect to $\nu$ with $d \eta / d \nu$ being (i) Lipschitz on each connected component of $M$ and (ii) strictly positive on $\cap_{i=0}^{\infty} f^{-i} \stackrel{\circ}{M}$. Notice that measures on $M$ with Lipschitz $d \eta / d \nu$ correspond to measures on $\mathcal{M}$ having a Lipschitz density with respect to Liouville measure.

Standing hypotheses for Theorems 1-3: We assume

(1) $f: M \rightarrow M$ is the billiard map defined in Sect. 1.2,

(2) $\left\{q_{0}\right\}$ is an infinitesimal hole of either Type I or Type II, and

(3) $\sigma \in \Sigma_{h}\left(q_{0}\right)$ where $h>0$ is assumed to be sufficiently small.

Theorem 1. (Common escape rate). All initial distributions $\eta \in \mathcal{G}$ have a common escape rate $-\log \vartheta_{*}$ for some $\vartheta_{*}<1$; more precisely, for all $\eta \in \mathcal{G}, \vartheta(\eta)$ is well defined and is equal to $\vartheta_{*}$.

Theorem 2. (Common limiting distribution).

(a) For all $\eta \in \mathcal{G}$, the normalized surviving distributions $f_{*}^{n} \eta /\left|f_{*}^{n} \eta\right|$ converge weakly to a common conditionally invariant distribution $\mu_{*}$ with $\vartheta\left(\mu_{*}\right)=\vartheta_{*}$.

(b) In fact, for all $\eta \in \mathcal{G}$, there is a constant $c(\eta)>0$ s.t. $\vartheta_{*}^{-n} \stackrel{\circ}{f}_{*}^{n} \eta$ converges weakly to $c(\eta) \mu_{*}$.

Thus from an observational point of view, $-\log \vartheta_{*}$ is the escape rate and $\mu_{*}$ the physical measure for the leaky system $\left(f, M, H_{\sigma}\right)$.

Theorem 3. (Geometry of limiting distribution).

(a) $\mu_{*}$ is singular with respect to $\nu$;

(b) $\mu_{*}$ has strictly positive conditional densities on local unstable manifolds.

The precise meaning of the statement in part (b) of Theorem 3 is that there are countably many "patches" $\left(V_{i}, \mu_{i}\right), i=1,2, \ldots$, where for each $i$,

(i) $V_{i} \subset M$ is the union of a continuous family of unstable curves $\left\{\gamma^{u}\right\}$;

(ii) $\mu_{i}$ is a measure on $V_{i}$ whose conditional measures on $\left\{\gamma^{u}\right\}$ have strictly positive densities with respect to the Riemannian measures on $\gamma^{u}$;

(iii) $\mu_{i} \leq \mu_{*}$ for each $i$, and $\sum_{i} \mu_{i} \geq \mu_{*}$.

This justifies viewing $\mu_{*}$ as the SRB measure for the leaky system $\left(f, M, H_{\sigma}\right)$.

Our final result can be interpreted as a kind of stability for the natural invariant measure $\nu$ of the billiard map without holes. 
Theorem 4. (Small-hole limit). We assume (1) and (2) in the Standing Hypotheses above. Let $\sigma_{h} \in \Sigma_{h}\left(q_{0}\right), h>0$, be an arbitrary family of holes, and let $-\log \vartheta_{*}\left(\sigma_{h}\right)$ and $\mu_{*}\left(\sigma_{h}\right)$ be the escape rate and physical measure for the leaky system $\left(f, M, H_{\sigma_{h}}\right)$. Then $\vartheta_{*}\left(\sigma_{h}\right) \rightarrow 1$ and $\mu_{*}\left(\sigma_{h}\right) \rightarrow \nu$ as $h \rightarrow 0$.

Some straightforward generalizations: Our proofs continue to hold under the more general conditions below, but we have elected not to discuss them (or to include them formally in the statement of our theorems) because keeping track of an increased number of objects will necessitate more cumbersome notation.

1. Holes. Our results apply to more general classes of holes than those described above. For example, we could fix a finite number of infinitesimal holes $\left\{q_{0}\right\}, \ldots,\left\{q_{k}\right\}$ and consider $\sigma=\cup_{i} \sigma_{i}$ with $\sigma_{i} \in \Sigma_{h}\left(q_{i}\right)$. In fact, we may take more than one $\sigma_{i}$ in each $\Sigma_{h}\left(q_{i}\right)$ for as long as the total number of holes is uniformly bounded. See Sect. 3.4 for further generalizations on the types of holes allowed.

2. Initial distributions. Theorems 1 and 2 (and consequently Theorems 3 and 4) remain true with $\mathcal{G}$ replaced by a broader class of measures. For example, we use only the Lipschitz property of $d \eta / d \nu$ along unstable leaves, and it is sufficient for $d \eta / d \nu$ to be strictly positive on large enough open sets (see Remark 6.3). Moreover, $d \eta / d \nu$ need not be bounded provided it blows up sufficiently slowly near the singularity set for $f$. Finally, we remark that Theorem 2(b) continues to hold without requiring that $d \eta / d \nu$ be strictly positive anywhere, except that now $c(\eta)$ might be 0 .

\section{Relevant Dynamical Structures}

Our plan is to show that the billiard maps described in Sect. 1.2 admit certain structures called "generalized horseshoes" which can be arranged to "respect the holes." The main results are summarized in Proposition 2.2 in Sect. 2.2 and proved in Sect. 3.

\subsection{Generalized horseshoes}

We begin by recalling the idea of a horseshoe with infinitely many branches and variable return times introduced in $[\mathrm{Y}]$ for general dynamical systems without holes. These objects will be referred to in this paper as "generalized horseshoes".

Following the notation in Sect. 1.1 of $[\mathrm{Y}]$, we consider a smooth or piecewise smooth invertible map $f: M \rightarrow M$, and let $\mu$ and $\mu_{\gamma}$ denote respectively the Riemannian measure on $M$ and on $\gamma$ where $\gamma \subset M$ is a submanifold. We say the pair $(\Lambda, R)$ defines a generalized horseshoe if $(\mathbf{P} 1)-(\mathbf{P} 5)$ below hold (see $[\mathrm{Y}]$ for precise formulation):

(P1) $\Lambda$ is a compact subset of $M$ with a hyperbolic product structure, i.e., $\Lambda=\left(\cup \Gamma^{u}\right) \cap\left(\cup \Gamma^{s}\right)$ where $\Gamma^{s}$ and $\Gamma^{u}$ are continuous families of local stable and unstable manifolds, and $\mu_{\gamma}\{\gamma \cap \Lambda\}>0$ for every $\gamma \in \Gamma^{u}$.

(P2) $R: \Lambda \rightarrow \mathbb{Z}^{+}$is a return time function to $\Lambda$. Modulo a set of $\mu$-measure zero, $\Lambda$ is the disjoint union of $s$-subsets $\Lambda_{j}, j=1,2, \cdots$, with the property that for each $j$, $\left.R\right|_{\Lambda_{j}}=R_{j} \in \mathbb{Z}^{+}$and $f^{R_{j}}\left(\Lambda_{j}\right)$ is a $u$-subset of $\Lambda$. 
There is a notion of separation time $s_{0}(\cdot, \cdot)$, depending only on the unstable coordinate, defined for pairs of points in $\Lambda$, and there are numbers $C>0$ and $\alpha<1$ such that the following hold for all $x, y \in \Lambda$ :

(P3) For $y \in \gamma^{s}(x), d\left(f^{n} x, f^{n} y\right) \leq C \alpha^{n}$ for all $n \geq 0$.

(P4) For $y \in \gamma^{u}(x)$ and $0 \leq k \leq n<s_{0}(x, y)$,

(a) $d\left(f^{n} x, f^{n} y\right) \leq C \alpha^{s_{0}(x, y)-n}$

(b) $\log \prod_{i=k}^{n} \frac{\operatorname{det} D f^{u}\left(f^{i} x\right)}{\operatorname{det} D f^{u}\left(f^{i} y\right)} \leq C \alpha^{s_{0}(x, y)-n}$.

(P5) (a) For $y \in \gamma^{s}(x), \log \Pi_{i=n}^{\infty} \frac{\operatorname{det} D f^{u}\left(f^{i} x\right)}{\operatorname{det} D f^{u}\left(f^{i} y\right)} \leq C \alpha^{n}$ for all $n \geq 0$.

(b) For $\gamma, \gamma^{\prime} \in \Gamma^{u}$, if $\Theta: \gamma \cap \Lambda \rightarrow \gamma^{\prime} \cap \Lambda$ is defined by $\Theta(x)=\gamma^{s}(x) \cap \gamma^{\prime}$, then $\Theta$ is absolutely continuous and $\frac{d\left(\Theta_{*}^{-1} \mu_{\gamma^{\prime}}\right)}{d \mu_{\gamma}}(x)=\Pi_{i=0}^{\infty} \frac{\operatorname{det} D f^{u}\left(f^{i} x\right)}{\operatorname{det} D f^{u}\left(f^{i} \Theta x\right)}$.

The meanings of the last three conditions are as follows: Orbits that have not "separated" are related by local hyperbolic estimates; they also have comparable derivatives. Specifically, (P3) and (P4)(a) are (nonuniform) hyperbolic conditions on orbits starting from $\Lambda$. (P4)(b) and (P5) treat more refined properties such as distortion and absolute continuity of $\Gamma^{s}$, conditions that are known to hold for $C^{1+\varepsilon}$ hyperbolic systems.

We say the generalized horseshoe $(\Lambda, R)$ has exponential return times if there exist $C_{0}>0$ and $\theta_{0}>0$ such that for all $\gamma \in \Gamma^{u}, \mu_{\gamma}\{R>n\} \leq C_{0} \theta_{0}^{n}$ for all $n \geq 0$.

The setting described above is that of $[\mathrm{Y}]$; it does not involve holes. In this setting, we now identify a set $H \subset M$ (to be regarded later as the hole) and introduce a few relevant terminologies. Let $(\Lambda, R)$ be a generalized horseshoe for $f$ with $\Lambda \subset(M \backslash H)$.

We say $(\Lambda, R)$ respects $H$ if for every $i$ and every $\ell$ with $0 \leq \ell \leq R_{i}, f^{\ell}\left(\Lambda_{i}\right)$ either does not intersect $H$ or is completely contained in $H$.

The following definitions of "mixing" are motivated by Markov-chain considerations: Let $\Lambda^{s} \subset \Lambda$ be an $s$-subset. We say $\Lambda^{s}$ makes a full return to $\Lambda$ at time $n$ if there are numbers $i_{0}, i_{1}, \cdots, i_{k}$ with $n=R_{i_{0}}+\cdots+R_{i_{k}}$ such that $\Lambda^{s} \subset \Lambda_{i_{0}}, f^{R_{i_{0}}+\cdots+R_{i_{j}}}\left(\Lambda^{s}\right) \subset \Lambda_{i_{j+1}}$ for $j<k$, and $f^{n}\left(\Lambda^{s}\right)$ is a $u$-subset of $\Lambda$. (i) We say the horseshoe $(\Lambda, R)$ is mixing if there exists $N$ such that for every $n \geq N$, some $s$-subset $\Lambda^{s}(n)$ makes a full return at time $n$. (ii) If $(\Lambda, R)$ respects $H$, then when we treat $H$ as a hole, we say the surviving dynamics are mixing if in addition to the condition in (i), we require that $f^{\ell} \Lambda^{s}(n) \cap H=\emptyset$ for all $\ell$ with $0 \leq \ell \leq n$. This is equivalent to requiring that $\Lambda^{s}(n)$ makes a full return to $\Lambda$ at time $n$ under the dynamics of $\stackrel{\circ}{f}$, where $f$ is the map defined in Sect. 1.1 .

We note that the mixing of $f$ in the usual sense of ergodic theory does not imply that any generalized horseshoe constructed is necessarily mixing in the sense of the last paragraph, nor does mixing of the horseshoe imply that of its surviving dynamics.

\subsection{Main Proposition for billiards with holes}

With these general ideas out of the way, we now return to the setting of the present paper. From here on, $f: M \rightarrow M$ is the billiard map of the 2-D Lorentz gas as in Sect. 1.2. The following result lies at the heart of the approach taken in this paper: 
Proposition 2.1. (Theorem 6(a) of $[\mathrm{Y}]$ ) The map $f$ admits a generalized horseshoe with exponential return times.

A few more definitions are needed before we are equipped to state our main proposition: We call $Q \subset M$ a rectangular region if $\partial Q=\partial^{u} Q \cup \partial^{s} Q$ where $\partial^{u} Q$ consists of two unstable curves and $\partial^{s} Q$ two stable curves. We let $Q(\Lambda)$ denote the smallest rectangular region containing $\Lambda$, and define $\mu^{u}(\Lambda):=\inf _{\gamma \in \Gamma^{u}} \mu_{\gamma}(\Lambda \cap \gamma)$. Finally, for a generalized horseshoe $(\Lambda, R)$ respecting a hole $H$, we define

$$
n(\Lambda, R ; H)=\sup \left\{n \in \mathbb{Z}^{+}: \text {no point in } \Lambda \text { falls into } H \text { in the first } n \text { iterates }\right\} .
$$

In the rest of this paper, $C$ and $\alpha$ will be the constants in (P3)-(P5) for the closed system $f$. All notation is as in Sect. 1.2.

Proposition 2.2. Given an infinitesimal hole $\left\{q_{0}\right\}$ of Type I or II, there exist $C_{0}, \kappa>0$, $\theta_{0} \in(0,1)$, and a rectangular region $Q$ such that for all small enough $h$ we have the following:

(a) For each $\sigma \in \Sigma_{h}\left(q_{0}\right)$,

(i) $f$ admits a generalized horseshoe $\left(\Lambda^{(\sigma)}, R^{(\sigma)}\right)$ respecting $H_{\sigma}$;

(ii) both $\left(\Lambda^{(\sigma)}, R^{(\sigma)}\right)$ and the corresponding surviving dynamics are mixing.

(b) All $\sigma \in \Sigma_{h}\left(q_{0}\right)$ have the following uniform properties:

(i) $Q\left(\Lambda^{(\sigma)}\right) \approx Q^{1}$, and $\mu^{u}\left(\Lambda^{(\sigma)}\right) \geq \kappa$;

(ii) $\mu_{\gamma}\left\{R^{(\sigma)}>n\right\}<C_{0} \theta_{0}^{n}$ for all $n \geq 0$;

(iii) (P3)-(P5) hold with the constants $C$ and $\alpha$.

Moreover, if $\bar{n}(h)=\inf _{\sigma \in \Sigma_{h}\left(q_{0}\right)} n\left(\Lambda, R ; H_{\sigma}\right)$, then $\bar{n}(h) \rightarrow \infty$ as $h \rightarrow 0$.

\section{Clarification:}

1. Here and in Sect. 3, there is a set, namely $H_{\sigma}$, that is identified to be "the hole," and a horseshoe is constructed to respect it. Notice that the construction is continued after a set enters $H_{\sigma}$. For reasons to become clear in Sect. 6, we cannot simply disregard those parts of the phase space that lie in the forward images of $H_{\sigma}$.

2. Proposition 2.2 treats only small $h$, i.e. small holes. The smallness of the holes and the uniformness of the estimates in part (b) are needed for the spectral arguments in Sect. 4 to apply. Without any restriction on $h$, all the conclusions of Proposition 2.2 remain true except for the following: (a)(ii), where for large holes the surviving dynamics need not be mixing, (b)(i), and (b)(ii), where $C_{0}$ and $\theta_{0}$ may be $\sigma$-dependent. The assertions for large $h$ will be evident from our proofs; no separate arguments will be provided.

A proof of Proposition 2.2 will require that we repeat the construction in the proof of Proposition 2.1 - and along the way, to carry out a treatment of holes and related issues. We believe it is more illuminating conceptually (and more efficient in terms of journal pages) to

\footnotetext{
${ }^{1}$ By $Q\left(\Lambda^{(\sigma)}\right) \approx Q$, we only wish to convey that both rectangular regions are located in roughly the same region of the phase space, $M$, and not anything technical in the sense of convergence.
} 
focus on what is new rather than to provide a proof written from scratch. We will, therefore, proceed as follows: The rest of this section contains a review of all the arguments used in the proof of Proposition 2.1, with technical estimates omitted and specific references given in their place. A proof of Proposition 2.2 is given in Sect. 3. There we go through the same arguments point by point, explain where modifications are needed and treat new issues that arise. For readers willing to skip more technical aspects of the analysis not related to holes, we expect that they will get a clear idea of the proof from this paper alone. For readers who wish to see all detail, we ask that they read this proof alongside the papers referenced.

\subsection{Outline of construction in $[\mathrm{Y}]$}

In this subsection, the setting and notation are both identical to that in Sect. 8 of [Y]. Referring the reader to $[Y]$ for detail, we identify below 7 main ideas that form the crux of the proof of Proposition 2.1. We will point out the use of billiard properties and other geometric facts that may potentially be impacted by the presence of holes. Holes are not discussed explicitly, however, until Sect. 3.

Notation and conventions: In $[\mathrm{Y}], S_{0}$ and $\partial M$ were used interchangeably. Here we use exclusively $\partial M$. Clearly, $f^{-1} \partial M$ is the discontinuity set of $f$.

(i) $u$ - and s-curves. Invariant cones $C^{u}$ and $C^{s}$ are fixed at each point, and curves all of whose tangent vectors are in $C^{u}$ (resp. $C^{s}$ ) are called $u$-curves (resp. $s$-curves).

(ii) The p-metric. Euclidean distance on $M$ is denoted by $d(\cdot, \cdot)$. Unless declared otherwise, distances and derivatives along $u$ - and $s$-curves are measured with respect to a semi-metric called the $p$-metric defined by $\cos \varphi d r$. These two metrics are related by $c p(x, y) \leq d(x, y) \leq$ $p(x, y)^{\frac{1}{2}}$. By $W_{\delta}^{u}(x)$, we refer to the piece of local unstable curve of $p$-length $2 \delta$ centered at $x$. (P3)-(P5) in Sect. 2.1 hold with respect to the $p$-metric. See Sect. 8.3 in [Y] for details.

(iii) Derivative bounds. With respect to the $p$-metric, there is a number $\lambda>1$ so that all vectors in $C^{u}$ are expanded by $\geq \lambda$ and all vectors in $C^{s}$ contracted by $\leq \lambda^{-1}$. Furthermore, derivatives at $x$ along $u$-curves are $\sim d(x, \partial M)^{-1}$. For purposes of distortion control, homogeneity strips of the form

$$
I_{k}=\left\{(r, \varphi): \frac{\pi}{2}-\frac{1}{k^{2}}<\varphi<\frac{\pi}{2}-\frac{1}{(k+1)^{2}}\right\}, \quad k \geq k_{0},
$$

are used, with $\left\{I_{-k}\right\}$ defined similarly in a neighborhood of $\varphi=-\frac{\pi}{2}$. For convenience, we will refer to $M \backslash\left(\cup_{|k| \geq k_{0}} I_{k}\right)$ as one of the " $I_{k}$ ".

Important Geometric Facts $(\dagger)$ : The following facts are used many times in the proof:

(a) the discontinuity set $f^{-1} \partial M$ is the union of a finite number of compact piecewise smooth decreasing curves, each of which stretches from $\{\varphi=\pi / 2\}$ to $\{\varphi=-\pi / 2\}$;

(b) $u$-curves are uniformly transversal (with angles bounded away from zero) to $\partial M$ and to $f^{-1} \partial M$.

1. Local stable and unstable manifolds. Only homogeneous local stable and unstable curves are considered. Homogeneity for $W_{\delta}^{u}$, for example, means that for all $n \geq 0, f^{-n} W_{\delta}^{u}$ 
lies in no more than 3 contiguous $I_{k}$. Let $\delta_{1}>0$ be a small number to be chosen. We let $\lambda_{1}=\lambda^{\frac{1}{4}}, \delta=\delta_{1}^{4}$, and define

$$
\begin{aligned}
& B_{\lambda_{1}, \delta_{1}}^{+}=\left\{x \in M: d\left(f^{n} x, \partial M \cup f^{-1}(\partial M)\right) \geq \delta_{1} \lambda_{1}^{-n} \text { for all } n \geq 0\right\} \\
& B_{\lambda_{1}, \delta_{1}}^{-}=\left\{x \in M: d\left(f^{-n} x, \partial M \cup f(\partial M)\right) \geq \delta_{1} \lambda_{1}^{-n} \text { for all } n \geq 0\right\}
\end{aligned}
$$

We require $d\left(f^{n} x, f^{-1}(\partial M)\right) \geq \delta_{1} \lambda_{1}^{-n}$ to ensure the existence of a local unstable curve through $x$, while the requirement on $d\left(f^{n} x, \partial M\right)$ is to ensure its homogeneity. ${ }^{2}$ Similar reasons apply to stable curves. Observe that (i) for all $x \in B_{\lambda_{1}, \delta_{1}}^{+}, W_{10 \delta}^{s}(x)$ is well defined and homogeneous (this is straightforward since $\delta<<\delta_{1}$ and $\lambda_{1}$ is closer to 1 than $\lambda$ ); and (ii) as $\delta_{1} \rightarrow 0, \nu\left(B_{\lambda_{1}, \delta_{1}}^{+}\right) \rightarrow 1$ (this follows from a standard Borel-Cantelli type argument). Analogous statements hold for $B_{\lambda_{1}, \delta_{1}}^{-}$.

2. Construction of the Cantor set $\Lambda$. The choice of $\Lambda$ is, in fact, quite arbitrary. We pick a density point $x_{1}$ of $B_{\lambda_{1}, 2 \delta_{1}}^{+} \cap B_{\lambda_{1}, 2 \delta_{1}}^{-}$at least $2 \delta_{1}$ away from $f^{-1}(\partial M) \cup \partial M \cup f(\partial M)$, and let $\Omega=W_{\delta}^{u}\left(x_{1}\right){ }^{3}$ For each $n$, we define

$$
\Omega_{n}=\left\{y \in \Omega: d\left(f^{i} y, f^{-1}(\partial M)\right) \geq \delta_{1} \lambda_{1}^{-i} \text { for } 0 \leq i \leq n\right\},
$$

and let $\Omega_{\infty}=\cap_{n} \Omega_{n}$. Then $\Omega_{\infty} \subset B_{\lambda_{1}, \delta_{1}}^{+}$, by the footnote in item 1 above and our choice of $x_{1}$ far from $\partial M$. Let $\Gamma^{s}$ consist of all $W_{\delta}^{s}(y), y \in \Omega_{\infty}$, and let $\Gamma^{u}$ be the set of all homogeneous $W_{\text {loc }}^{u}$ curves that meet every $\gamma^{s} \in \Gamma^{s}$ and which extend by a distance $>\delta$ on both sides of the curves in $\Gamma^{s}$. The set $\Lambda$, which is defined to be $\left(\cup \Gamma^{u}\right) \cap\left(\cup \Gamma^{s}\right)$, clearly has a hyperbolic product structure. (P5)(b) is standard. This together with the choice of $x_{1}$ guarantees $\mu_{\gamma}\{\gamma \cap \Lambda\}>0$ for $\gamma \in \Gamma^{u}$, completing the proof of (P1).

A natural definition of separation time for $x, y \in \gamma^{u}$ is as follows: Let $[x, y]$ be the subsegment of $\gamma^{u}$ connecting $x$ and $y$. Then $f^{n} x$ and $f^{n} y$ are "not yet separated," i.e. $s_{0}(x, y) \geq n$, if for all $i \leq n, f^{i}[x, y]$ is connected and is contained in at most 3 contiguous $I_{k}$. With this definition of $s_{0}(\cdot, \cdot),(\mathbf{P} 3)-(\mathbf{P} 5)(\mathbf{a})$ are checked using previously known billiard estimates.

3. The return map $f^{R}: \Lambda \rightarrow \Lambda$. We point out that there is some flexibility in choosing the return map $f^{R}$ : Certain conditions have to be met when a return takes place, but when these conditions are met, we are not obligated to call it a return; in particular, $R$ is not necessarily the first time an $s$-subrectangle of $Q u$-crosses $Q$ where $Q=Q(\Lambda)$.

We first define $f^{R}$ on $\Omega_{\infty}$. Let $\tilde{\Omega}_{n}=\Omega_{n} \backslash\{R \leq n\}$. On $\tilde{\Omega}_{n}$ is a partition $\tilde{\mathcal{P}}_{n}$ whose elements are segments representing distinct trajectories. The rules are different before and after a certain time $R_{1}$, a lower bound for which is determined by $\lambda_{1}, \delta_{1}$ and the derivative of $f .4$

\footnotetext{
${ }^{2}$ In fact, provided $\delta_{1}$ is chosen sufficiently small, one can verify that $d\left(f^{n} x, f^{-1}(\partial M)\right) \geq \delta_{1} \lambda_{1}^{-n}$ implies that $d\left(f^{n+1} x, \partial M\right) \geq \delta_{1} \lambda_{1}^{-(n+1)}$ for all $n \geq 0$. This fact, which was not used in [Y], will be used in item 2 below to simplify our presentation.

${ }^{3}$ Later we will impose one further technical condition on the choice of $x_{1}$. See the very end of Sect. 2.4.

${ }^{4}$ In [Y], properties of $R_{1}$ are used in 4 places: (I)(i) in Sect. 3.2, Sublemma 3 in Sect. 7.3, the paragraph following $(* *)$ in Sect. 8.4, and a requirement in Sect. 8.3 that stable manifolds pushed forward more than $R_{1}$ times are sufficiently contracted.
} 
(a) For $n<R_{1}, \tilde{\mathcal{P}}_{n}$ is constructed from the results of the previous step $^{5}$ as follows: Let $\omega \in \tilde{\mathcal{P}}_{n-1}$, and let $\omega^{\prime}$ be a component of $\omega \cap \Omega_{n}$. Inserting cut-points only where necessary, we divide $\omega^{\prime}$ into subsegments $\omega_{i}$ with the property that $f^{n}\left(\omega_{i}\right)$ is homogeneous. These are the elements of $\tilde{\mathcal{P}}_{n}$. No point returns before time $R_{1}$.

(b) For $n \geq R_{1}$, we proceed as in (a) to obtain $\omega_{i}$. If $f^{n}\left(\omega_{i}\right) u$-crosses the middle of $Q$ with $\geq 1.5 \delta$ sticking out on each side, then we declare that $R=n$ on $\omega_{i} \cap f^{-n} \Lambda$, and the elements of $\left.\tilde{\mathcal{P}}_{n}\right|_{\omega_{i} \cap \tilde{\Omega}_{n}}$ are the connected components of $\omega_{i} \backslash f^{-n} \Lambda$. Otherwise put $\omega_{i} \in \tilde{\mathcal{P}}_{n}$ as before.

This defines $R$ on a subset of $\Omega_{\infty}$ (which we do not know yet has full measure); the definition is extended to the associated $s$-subset of $\Lambda$ by making $R$ constant on $W_{\text {loc }}^{s}$-curves. The $s$-subsets associated with $\omega_{i} \cap f^{-n} \Lambda$ in (b) above are the $\Lambda_{j}$ in (P2). It remains to check that $f^{R}\left(\Lambda_{j}\right)$ is in fact a $u$-subset of $\Lambda$. This is called the "matching of Cantor sets" in $[\mathrm{Y}]$ and is a consequence of the fact that $\Omega_{\infty}$ is dynamically defined and that $R_{1}$ is chosen sufficiently large.

It remains to prove that $p\{R \geq n\}$ decays exponentially with $n$. Paragraphs 4,5 and 6 contain the 3 main ingredients of the proof, with the final count given in 7 .

4. Growth of $u$-curves to "long" segments. This is probably the single most important point, so we include a few more details. We first give the main idea before adapting it to the form it is used. Let $\varepsilon_{0}>0$ be a number the significance of which we will explain later. Here we think of a $u$-curve whose $p$-length exceeds $\varepsilon_{0}>0$ as "long". Consider a $u$-curve $\omega$. We introduce a stopping time $T$ on $\omega$ as follows. For $n=1,2, \cdots$, we divide $f^{n} \omega$ into homogeneous segments representing distinguishable trajectories. For $x \in \omega$, let

$$
T(x)=\inf \left\{n>0: \text { the segment of } f^{n} \omega \text { containing } f^{n} x \text { has } p \text {-length }>\varepsilon_{0}\right\} .
$$

Lemma 2.3. There exist $D_{1}>0$ and $\theta_{1}<1$ such that for any $u$-curve $\omega$,

$$
p(\omega \backslash\{T \leq n\})<D_{1} \theta_{1}^{n} \quad \text { for all } n \geq 1 .
$$

This lemma relies on the following important geometric property of the class of billiards in question. This choice of $\varepsilon_{0}>0$ is closely connected to this property:

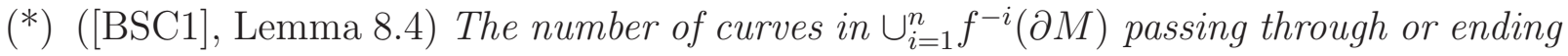
in any one point in $M$ is $\leq K_{0} n$, where $K_{0}$ is a constant depending only on the "table" $X$.

Let $\alpha_{0}:=2 \sum_{k=k_{0}}^{\infty} \frac{1}{k^{2}}$ where $\left\{I_{k},|k| \geq k_{0}\right\}$ are the homogeneity strips, and assume that $\lambda^{-1}+\alpha_{0}<1$. Choose $m$ large enough that $\theta_{1}:=\left(K_{0} m+1\right)^{\frac{1}{m}}\left(\lambda^{-1}+\alpha_{0}\right)<1$. We may then fix $\varepsilon_{0}<\delta$ to be small enough that every $W_{\text {loc }}^{u}$-curve of $p$-length $\leq \varepsilon_{0}$ has the property that it intersects $\leq K_{0} m$ smooth segments of $\cup_{1}^{m} f^{-i}(\partial M)$, so that the $f^{m}$-image of such a $W_{\text {loc }}^{u}$-curve has $\leq\left(K_{0} m+1\right)$ connected components.

\footnotetext{
${ }^{5}$ In $[Y]$, it was sufficient to allow returns to $\Lambda$ at times that were multiples of a large fixed integer $m$. Not only is this not necessary (see Paragraph 4), here it is essential that we avoid such periodic behavior to ensure mixing. Thus we take $m=1$ when choosing return times in Paragraph 3 . This is the only substantial departure we make from the construction in $[\mathrm{Y}]$.
} 
The proof of Lemma 2.3, which follows [BSC2], goes as follows: Consider a large $n$, which we may assume is a multiple of $m$. (Once Lemma 2.3 is proved for multiples of $m$, the estimate can be extended to intermediate values by enlarging the constant $D_{1}$.) We label distinguishable trajectories by their $I_{k}$-itineraries. Notice that because $f^{i} \omega$ is the union of a number of (disconnected) $u$-curves, it is possible for many distinguishable trajectories to have the same $I_{k}$-itinerary. Specifically, by $(*)$, each trajectory of length $j m, j \in \mathbb{Z}^{+}$, gives birth to at most $\left(K_{0} m+1\right)$ trajectories of length $(j+1) m$ with the same $I_{k}$-itinerary. To estimate $p(\omega \backslash\{T \leq n\})$, we assume the worst case scenario, in which the $f^{n}$-images of subsegments of $\omega$ corresponding to all distinguishable trajectories have length $\leq \varepsilon_{0}$. We then sum over all possible itineraries using bounds on $D f$ along $u$-curves in $I_{k}$.

We now adapt Lemma 2.3 to the form in which it will be used. Let $\omega=f^{k} \omega^{\prime}$ for some $\omega^{\prime} \in \tilde{\mathcal{P}}_{k}$ in the construction in Paragraph 3. As we continue to evolve $\omega, f^{n} \omega$ is not just chopped up by the discontinuity set, bits of it that go near $f^{-1}(\partial M)$ will be lost by intersecting with $f^{k+n} \Omega_{k+n}$, and we need to estimate $p\left(\omega_{n} \backslash\{T \leq n\}\right)$ where $\omega_{n}:=$ $\omega \cap f^{k}\left(\Omega_{k+n}\right)$ takes into consideration these intersections and $T$ is redefined accordingly. $A$ priori this may require a larger bound than that given in Lemma 2.3: it is conceivable that there are segments that will grow to length $\varepsilon_{0}$ without losing these "bits" but which do not now reach this reference length. We claim that all such segments have been counted, because (i) the deletion procedure does not create new connected components; it merely trims the ends of segments adjacent to cut-points; and (ii) the combinatorics in Lemma 2.1 count all possible itineraries (and not just those that lead to "short" segments). This yields the desired estimate on $p\left(\omega_{n} \backslash\{T \leq n\}\right)$, which is Sublemma 2 in Sect. 8.4 of [Y].

5. Growth of "gaps" of $\Lambda$. Let $\omega$ be the subsegment of some $\gamma^{u} \in \Gamma^{u}$ connecting the two $s$-boundaries of $Q$. We think of this as a return in the construction outlined in Paragraph 3 , with the connected components $\omega^{\prime}$ of $\omega^{c}=\omega \backslash \Lambda$ being $f^{k}$-images of elements of $\tilde{\mathcal{P}}_{k}$. We define a stopping time $T$ on $\omega^{c}$ by considering one $\omega^{\prime}$ at a time and defining on it the stopping time in Paragraph 4.

Lemma 2.4. There exist $D_{2}>0$ and $\theta_{2}<1$ independent of $\omega$ such that

$$
p\left(\omega_{n}^{c} \backslash\{T \leq n\}\right)<D_{2} \theta_{2}^{n} \quad \text { for all } n \geq 1 .
$$

The idea of the proof is as follows. We may identify $\omega$ with $\Omega$ (see Paragraph 2), so that the collection of $\omega^{\prime}$ is precisely the collection of gaps in $\Lambda$. We say $\omega^{\prime}$ is of generation $q$ if this is the first time a part of $\omega^{\prime}$ is removed in the construction of $\Omega_{\infty}$. There are two separate estimates:

$$
(I):=\sum_{q>\varepsilon n} \sum_{\operatorname{gen}\left(\omega^{\prime}\right)=q} p\left(\omega^{\prime}\right) ; \quad(I I):=\sum_{q \leq \varepsilon n} \sum_{\operatorname{gen}\left(\omega^{\prime}\right)=q} p\left(\omega_{n}^{\prime} \backslash\{T \leq n\}\right) .
$$

(I) has exponentially small $p$-measure: this follows from a comparison of the growth rate of $D f$ along $u$-curves versus the rate at which these curves get cut (see Paragraph 4). (II) is bounded above by

$$
\sum_{q \leq \varepsilon n} \sum_{\operatorname{gen}\left(\omega^{\prime}\right)=q} \frac{C p\left(\omega^{\prime}\right)}{p\left(f^{q-1} \omega^{\prime}\right)} \cdot D_{1} \theta_{1}^{n-q-1} .
$$


This is obtained by applying the modified version of Lemma 2.3 to $f^{q-1} \omega^{\prime}$. A lower bound on $p\left(f^{q-1} \omega^{\prime}\right)$ can be estimated as these curves have not been cut by $f^{-1}(\partial M)$ (though they may have been shortened to maintain homogeneity), reducing the estimate to $\sum_{q} \sum_{\text {gen }\left(\omega^{\prime}\right)=q} p\left(\omega^{\prime}\right)$, which is $\leq p(\omega)$.

6. Return of "long" segments. This concerns the evolution of unstable curves after they have grown "long", where "long" has the same meaning as in Paragraph 4. The following geometric fact from [BSC2] is used:

(**) Given $\varepsilon_{0}>0, \exists n_{0}$ s.t. for every homogeneous $W_{\text {loc }}^{u}$-curve $\omega$ with $p(\omega)>\varepsilon_{0}$ and every $q \geq n_{0}$, fq $\omega$ contains a homogeneous segment which $u$-crosses the middle half of $Q$ with $>2 \delta$ sticking out from each side.

We choose $\varepsilon_{0}>0$ as explained in Paragraph 4 above, and apply $(* *)$ with $q=n_{0}$ to the segments that arise in Paragraphs 4 and 5 when the stopping time $T$ is reached. For example, $\omega$ here may be equal to $f^{n} \omega^{\prime \prime}$ where $\omega^{\prime \prime}$ is a subsegment of the $\omega$ in the last paragraph of Paragraph 4 with $\left.T\right|_{\omega^{\prime \prime}}=n$. We claim that a fixed fraction of such a segment will make a return within $n_{0}$ iterates. To guarantee that, two other facts need to be established: (i) The small bits deleted by intersecting with $f^{n+k} \Omega_{n+k}$ before the return still leave a segment which $u$-crosses the middle half of $Q$ with $>1.5 \delta$ sticking out from each side; this is easily checked. (ii) For $q \leq n_{0},\left(f^{q}\right)^{\prime}$ is uniformly bounded on $f^{-q}$-images of homogeneous segments that $u$-cross $Q$. This is true because a segment contained in $I_{k}$ for too large a $k$ cannot grow to length $\delta$ in $n_{0}$ iterates.

7. Tail estimate of return time. We now prove $p\{R \geq n\} \leq C_{0} \theta_{0}^{n}$ for some $\theta_{0}<1$. On $\Omega$, introduce a sequence of stopping times $T_{1}<T_{2}<\cdots$ as follows: A stopping time $T$ of the type in Paragraph 4 or 5 is initiated on a segment as soon as $T_{k}$ is reached, and $T_{k+1}$ is set equal to $T_{k}+T$. In this process, we stop considering points that are lost to deletions or have returned to $\Lambda$. The desired bound follows immediately from the following two estimates:

(i) There exists $\varepsilon^{\prime}>0, D_{3} \geq 1$, and $\theta_{3}<1$ such that $p\left(T_{\left[\varepsilon^{\prime} n\right]}>n\right)<D_{3} \theta_{3}^{n}$.

(ii) There exists $\varepsilon_{1}>0$ such that if $T_{k} \mid \omega=n$, then $p\left(\omega \cap\left\{R>n+n_{0}\right\}\right) \leq\left(1-\varepsilon_{1}\right) p(\omega)$ where $n_{0}$ is as in $(* *)$ in Paragraph 6 .

(ii) is explained in Paragraph 6. To prove (i), we let $p=\left[\varepsilon^{\prime} n\right]$, decompose $\Omega$ into sets of the form $A\left(k_{1}, \cdots, k_{p}\right)=\left\{x \in \Omega: T_{1}(x), \cdots, T_{p}(x)\right.$ are defined with $\left.T_{i}=k_{i}\right\}$, apply Lemmas 2.1 and 2.2 to each set and recombine the results. The argument here is combinatorial, and does not use further geometric information about the system.

\subsection{Sketch of proof of (**) following [BSC2]}

Property $(* *)$ is a weaker version of Theorem 3.13 in [BSC2]. We refer the reader to [BSC2] for detail, but include an outline of its proof because a modified version of the argument will be needed in the proof of Proposition 2.2.

We omit the proof of the following elementary fact, which relies on the geometry of the discontinuity set including Property $(*)$ : 
Sublemma A. Given any $u$-curve $\gamma$, through $\mu_{\gamma}$-a.e. $x \in \gamma$ passes a homogeneous $W_{\delta(x)}^{s}(x)$ for some $\delta(x)>0$. The analogous statement holds for s-curves.

Instead of considering every $W_{\mathrm{loc}}^{u}$-curve as required in (**), the problem is reduced to a finite number of "mixing boxes" $U_{1}, U_{2}, \ldots, U_{k}$ with the following properties:

(i) $U_{j}$ is a hyperbolic product set defined by (homogeneous) families $\Gamma^{u}\left(U_{j}\right)$ and $\Gamma^{s}\left(U_{j}\right)$; located in the middle third of $U_{j}$ is an $s$-subset $\tilde{U}_{j}$ with $\nu\left(\tilde{U}_{j}\right)>0$;

(ii) $\cup \Gamma^{u}\left(U_{j}\right)$ fills up nearly $100 \%$ of the measure of $Q\left(U_{j}\right)$; and

(iii) every $W_{\text {loc }}^{u}$-curve $\omega$ with $p(\omega)>\varepsilon_{0}$ passes through the middle third of one of the $Q\left(U_{j}\right)$ in the manner shown in Fig. 1 (left).

That (i) and (ii) can be arranged follows from Sublemma A. That a finite number of $U_{j}$ suffices for (iii) follows from a compactness argument.

Next we choose a suitable subset $\tilde{U}_{0} \subset \Lambda$ to be used in the mixing. To do that, first pick a hyperbolic product set $U_{0}$ related to $Q(\Lambda)$ as shown in Fig. 1 (right). We require that it meet $Q(\Lambda)$ in a set of positive measure, that it sticks out of $Q(\Lambda)$ in the $u$-direction by more than $2 \delta$, and that the curves in $\Gamma^{u}\left(U_{0}\right)$ fill up nearly $100 \%$ of $Q\left(U_{0}\right)$. Let $\ell_{0}>0$ be a small number, and let $\tilde{U}_{0} \subset U_{0}$ consist of those density points of $U_{0} \cap Q(\Lambda)$ with the additional property that if a homogeneous stable curve $\gamma^{s}$ with $p\left(\gamma^{s}\right)<\ell_{0}$ meets such a point, then $p\left(\gamma^{s} \cap U_{0}\right) / p\left(\gamma^{s}\right) \approx 1$. For $\ell_{0}$ small enough, $\nu\left(\tilde{U}_{0}\right)>0$ because the foliation into $W_{\text {loc }}^{u}$-curves is absolutely continuous.
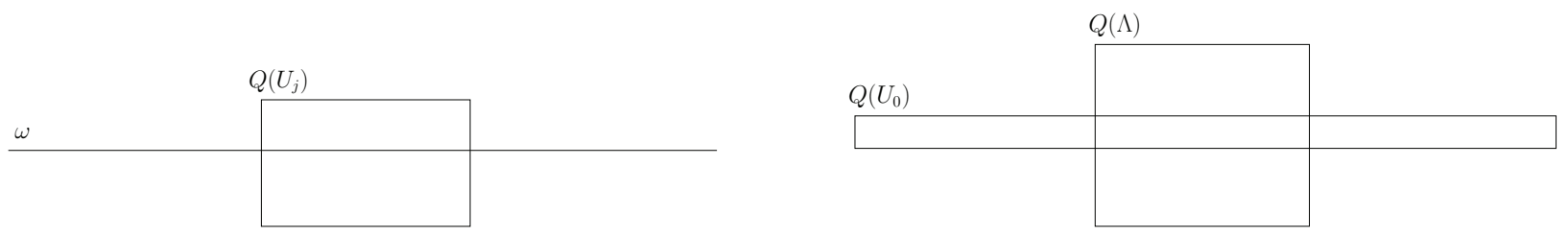

Figure 1: Left: A mixing box $U_{j}$. Right: The target box $U_{0}$.

By the mixing property of $(f, \nu)$, there exists $n_{0}$ such that for all $q \geq n_{0}, \nu\left(f^{q}\left(\tilde{U}_{j}\right) \cap \tilde{U}_{0}\right)>0$ for every $\tilde{U}_{j}$. We may assume also that $n_{0}$ is so large that for $q \geq n_{0}$, if $x \in \tilde{U}_{j}$ is such that $f^{q} x \in \tilde{U}_{0}$, then $p\left(f^{q}\left(\gamma^{s}(x)\right)\right)<\ell_{0}$ where $\gamma^{s}(x)$ is the stable curve in $\Gamma^{s}\left(\tilde{U}_{j}\right)$ passing through $x$. Let $q \geq n_{0}$ and $j$ be fixed, and let $x \in \tilde{U}_{j}$ be as above. From the high density of unstable curves in both $U_{j}$ and $U_{0}$, we are guaranteed that there are two elements $\gamma_{1}^{u}, \gamma_{2}^{u} \in \Gamma^{u}\left(U_{j}\right)$ sandwiching the middle third of $Q\left(U_{j}\right)$ such that for each $i$, a subsegment of $\gamma_{i}^{u}$ containing $\gamma^{s}(x) \cap \gamma_{i}^{u}$ is mapped under $f^{q}$ onto some $\hat{\gamma}_{i}^{u} \in \Gamma^{u}\left(U_{0}\right)$. Let $Q^{*}=Q^{*}(q, j)$ be the $u$ subrectangle of $Q\left(U_{0}\right)$ with $\partial^{u} Q^{*}=\hat{\gamma}_{1}^{u} \cup \hat{\gamma}_{2}^{u}$.

Sublemma B. $\left.f^{-q}\right|_{Q^{*}}$ is continuous, equivalently, $Q^{*} \cap\left(\cup_{0}^{q} f^{i}(\partial M)\right)=\emptyset$.

Sublemma B is an immediate consequence of the geometry of the discontinuity set: By the choice of $x_{1}$ in item 2 of Sect. 2.3, $Q^{*} \cap \partial M=\emptyset$. Suppose $Q^{*} \cap\left(\cup_{1}^{q} f^{i}(\partial M)\right) \neq \emptyset$. Since $\cup_{1}^{q} f^{i}(\partial M)$ is the union of finitely many piecewise smooth (increasing) u-curves each connected component of which stretches from $\{\varphi=-\pi / 2\}$ to $\{\varphi=\pi / 2\}$, and these curves cannot touch $\partial^{u} Q^{*}$, a piecewise smooth segment from $\cup_{1}^{q} f^{i}(\partial M)$ that enters $Q^{*}$ through one 
component of $\partial^{s} Q^{*}$ must exit through the other. In particular, it must $\operatorname{cross} f^{q} \gamma^{s}(x)$, which is a contradiction.

To prove $\left({ }^{* *}\right)$, let $\omega$ be a $W_{\text {loc }}^{u}$-curve with $p(\omega)>\varepsilon_{0}$. We pick $U_{j}$ so that $\omega$ passes through the middle third of $U_{j}$ as in (iii) above. Sublemma B then guarantees that $f^{q}\left(\omega \cap f^{-q} Q^{*}\right)$ connects the two components of $\partial^{s} Q^{*}$. This completes the proof of $\left({ }^{*}\right)$, except that we have not yet verified that $f^{q}\left(\omega \cap f^{-q} Q^{*}\right)$ is homogeneous.

To finish this last point, we modify the above argument as follows: First, we define a $W_{\text {loc }}^{u}$ curve $\gamma$ to be strictly homogeneous if for all $n \geq 0, f^{-n} \gamma$ is contained inside one homogeneity strip $I_{k}(n)$. Strict homogeneity for $W_{\text {loc }}^{s}$ curves is defined analogously. The conclusions of Sublemma A remain valid if, in its statement, the word "homogeneous" is replaced by "strictly homogeneous." Thus the mixing boxes $U_{1}, \ldots, U_{k}$ can be chosen so that their defining families are comprised entirely of strictly homogeneous local manifolds. Furthermore, if $x_{1}$ is also chosen as a density point of points with sufficiently long strictly homogeneous unstable curves, $\Gamma^{u}\left(U_{0}\right)$ can be chosen to be comprised entirely of strictly homogeneous $W_{\text {loc }}^{u}$ curves. Having done this, an argument very similar to the proof of Sublemma B shows that $f^{-i} Q^{*} \cap\left(\cup_{k} \partial I_{k}\right)=\emptyset$ for $0 \leq i \leq q$, and this completes the proof of $\left(^{* *}\right)$.

\section{$3 \quad$ Horseshoes Respecting Holes for Billiard Maps}

\subsection{Geometry of holes in phase space}

We summarize here some relevant geometric properties and explain how we plan to incorporate holes into our horseshoe construction.

Holes of Type I. Recall from Sect. 1.2 that for $q_{0} \in \cup \Gamma_{i}$ and $\sigma \in \Sigma_{h}\left(q_{0}\right), H_{\sigma} \subset M$ is a rectangle of the form $(a, b) \times\left[-\frac{\pi}{2}, \frac{\pi}{2}\right]$. We define $\partial H_{\sigma}:=\{a, b\} \times\left[-\frac{\pi}{2}, \frac{\pi}{2}\right]$, i.e. $\partial H_{\sigma}$ is the boundary of $H_{\sigma}$ viewed as a subset of $M$. It will also be convenient to let $H_{0} \subset M$ denote the vertical line $\left\{q_{0}\right\} \times\left[-\frac{\pi}{2}, \frac{\pi}{2}\right]$. To construct a horseshoe respecting $H_{\sigma}$, it is necessary to view two nearby points as having separated when they lie on opposite sides of $\partial H_{\sigma}$ or on opposite sides of $H_{\sigma}$ in $M \backslash H_{\sigma}$. Thus it is convenient to view $f^{-1}\left(\partial H_{\sigma}\right)$ as part of the discontinuity set of $f$. For simplicity, consider first the case where $q_{0}$ does not lie on a line in the table $\mathrm{X}$ tangent to more than one scatterer. Then $f^{-1}\left(\partial H_{\sigma}\right)$ is a finite union of pairs of roughly parallel, smooth $s$-curves. (Recall that $s$-curves are negatively sloped, with slopes uniformly bounded away from 0 and $-\infty$.) Each of the curves comprising $f^{-1}\left(\partial H_{\sigma}\right)$ begins and ends in $\partial M \cup f^{-1}(\partial M)$, that is to say, the geometric properties of $f^{-1}\left(\partial H_{\sigma}\right) \cup f^{-1}(\partial M)$ are similar to those of $f^{-1}(\partial M)$. Likewise, $f\left(\partial H_{\sigma}\right)$ is a finite of union of pairs of (increasing) $u$-curves that begin and end in $\partial M \cup f(\partial M)$, and it will be convenient to regard that as part of the discontinuity set of $f^{-1}$.

Let $N_{\varepsilon}(\cdot)$ denote the $\varepsilon$-neighborhood of a set. We will need the following lemma.

Lemma 3.1. For each $\varepsilon>0$ there is an $h>0$ such that for each $\sigma \in \Sigma_{h}, H_{\sigma} \subset N_{\varepsilon}\left(H_{0}\right)$, $f H_{\sigma} \subset N_{\varepsilon}\left(f H_{0}\right)$, and $f^{-1} H_{\sigma} \subset N_{\varepsilon}\left(f^{-1} H_{0}\right)$.

As $f$ is discontinuous, Lemma 3.1 is not immediate. However, it can be easily verified, and we leave the proof to the reader. 
Points $q_{0}$ that lie on lines in $X$ with multiple tangencies to scatterers lead to slightly more complicated geometries, and special care is needed when defining what is meant by $f H_{0}$ and $f^{-1} H_{0}$. For example, consider the case where $q_{0} \in \Gamma_{3}$ lies on a line that is tangent to $\Gamma_{1}$ and $\Gamma_{2}$, but which is not tangent to any other scatterer including $\Gamma_{3}$. Suppose further that $r_{1} \in \Gamma_{1}, r_{2} \in \Gamma_{2}$ are the points of tangency, that $r_{2}$ is closer to $q_{0}$ than $r_{1}$ is, that no other scatterer touches the line segment $\left[q_{0}, r_{1}\right]$, and that $\Gamma_{1}$ and $\Gamma_{2}$ both lie on the same side of $\left[q_{0}, r_{1}\right]$; see Fig. 2 (left). Let $\sigma$ be a small hole of Type I with $q_{0} \in \sigma$. Then in $\Gamma_{2} \times[-\pi / 2, \pi / 2], f^{-1}\left(\partial H_{\sigma}\right)$ appears as described above. However, $\Gamma_{2}$ "obstructs" the view of $\sigma$ from $\Gamma_{1}$, and so in $\Gamma_{1} \times[-\pi / 2, \pi / 2], f^{-1}\left(H_{\sigma}\right)$ is a small triangular region whose three sides are composed of a segment from $\Gamma_{1} \times\{\pi / 2\}$, a segment from $f^{-1}\left(\Gamma_{2} \times\{\pi / 2\}\right)$, and a single segment from $f^{-1}\left(\partial H_{\sigma}\right)$. See Fig. 2 (right). As a consequence, when we write $f^{-1} H_{0}$, we include in this set not just $\left(r_{2}, \pi / 2\right)$, but also $f^{-1}\left(r_{2}, \pi / 2\right)=\left(r_{1}, \pi / 2\right)$. This is necessary in order for Lemma 3.1 to continue to hold. Aside from such minor modifications, the case of multiple tangencies is no different than when they are not present, and we leave further details to the reader.
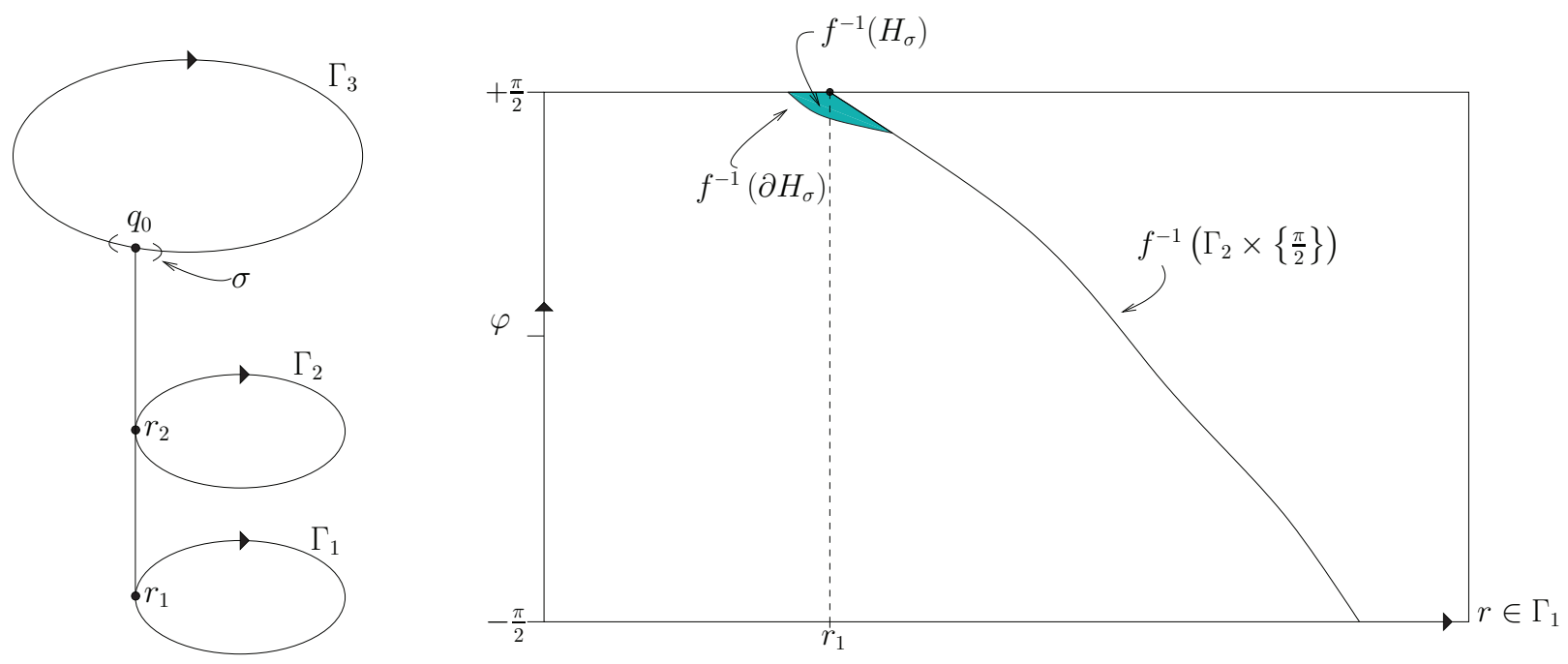

Figure 2: An infinitesmal hole aligned with multiple tangencies. Left: $q_{0}$ lies on a line segment in the billiard table $X$ that is tangent to two scatterers. Right: Induced singularity curves in the subset $\Gamma_{1} \times[-\pi / 2, \pi / 2]$ of the phase space $M$.

Holes of Type II. For simplicity, consider first the case where $q_{0}$ does not lie on a line in the "table" $X$ tangent to more than one scatterer. Recall from Sect. 1.2 that "the hole" $H_{\sigma}$ here is taken to be $f\left(B_{\sigma}\right)$ where $B_{\sigma}$ consists of points in $M$ which enter $\sigma \times \mathbb{S}^{1}$ under the billiard flow before returning to the section $M$. As with holes of Type I, we define $\partial H_{\sigma}$ to be the boundary of $H_{\sigma}$ viewed as a subset of $M$. The set $B_{\sigma}$ as a subset of $M$ has similar geometric properties as $f^{-1} H_{\sigma}$ for Type I holes, i.e., $f^{-1}\left(\partial H_{\sigma}\right) \backslash\left(\partial M \cup f^{-1}(\partial M)\right)$ consists of pairs of negatively sloped curves ending in $\partial M \cup f^{-1}(\partial M)$. The slopes of these curves are uniformly bounded (independent of $\sigma$ ) away from $-\infty$ and 0 . For the reasons discussed, it will be convenient to view this set as part of the discontinuity set of $f$. The infinitesimal hole $H_{0} \subset M$ is defined in the natural way, and the analog of Lemma 3.1 can be verified. We will say more about the geometry of $H_{\sigma}$ in Sect. 3.3. 
Points $q_{0}$ that lie on multiple tangencies lead to slightly more complicated geometries, and special care is needed when defining what is meant by the sets $f^{-1} H_{0}, H_{0}$, and $f H_{0}$ as in the case of Type I holes.

Further generalizations on holes of Type II: In addition to the generalizations discussed in Sect. 1.3, sufficient conditions on the holes allowed in $\Sigma_{h}$ for Prop. 2.2 to remain true are the following, as can be seen from our proofs:

(1) There exist $N$ and $L$ for which the following hold for all sufficiently small $h$ :

(a) $f^{-1}\left(\partial H_{\sigma}\right), \partial H_{\sigma}$, and $f\left(\partial H_{\sigma}\right)$ each consist of no more than $N$ smooth curves, all of which have length no greater than $L$.

(b) For each $\sigma \in \Sigma_{h}, f^{-1}\left(\partial H_{\sigma}\right) \backslash\left(\partial M \cup f^{-1}(\partial M)\right)$ consists of piecewise smooth, negatively sloped curves (with slopes uniformly bounded away from $-\infty$ and 0 ), and the end points of these curves must lie on $\partial M \cup f^{-1}(\partial M)$.

(2) The analog of Lemma 3.1 holds.

Thus it would be permissible to allow a convex hole $\sigma$ to be in $\Sigma_{h}$ that $\operatorname{did}$ not have a $C^{3}$ simple closed curve with strictly positive curvature as its boundary. For example, conditions (a) and (b) above hold if $\partial \sigma$ is a piecewise $C^{3}$ simple closed curve which consists of finitely many smooth segments that are either strictly positively curved or flat. As another generalization, consider the case when any line segment in the table $X$ with its endpoints on two scatterers that passes through the convex hull of $\sigma$ also intersects $\sigma$. Then it is no loss of generality to replace $\sigma$ by its convex hull. Using this, one can often verify that the set $H_{\sigma}$ that arises satisfies properties (a) and (b) above, even if $\sigma$ is not itself convex. See Fig. 3.
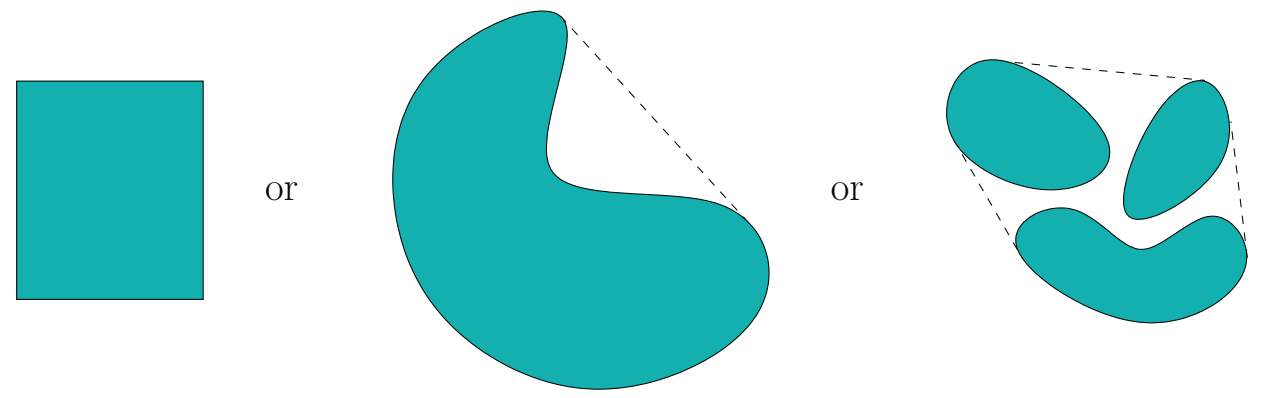

Figure 3: Examples of Type II holes that are permissible.

In Sect. 3.2, the discussion is for holes of Type I with a single interval deleted. The proof follows mutatis mutandis for holes of Type II, with the necessary minor modifications discussed in Sect. 3.3.

\subsection{Proof of Proposition 2.2 (for holes of Type I)}

The idea of the proof is as follows. First we construct a horseshoe $\left(\Lambda^{(0)}, R^{(0)}\right)$ with the desired properties for the infinitesimal hole $\left\{q_{0}\right\}$. Then we construct $\left(\Lambda^{(\sigma)}, R^{(\sigma)}\right)$ for all $\sigma \in \Sigma_{h}\left(q_{0}\right)$, and show that with $\Lambda^{(\sigma)}$ sufficiently close to $\Lambda^{(0)}$ in a sense to be made precise, $\left(\Lambda^{(\sigma)}, R^{(\sigma)}\right)$ will inherit the desired properties with essentially the same bounds. To ensure that $\Lambda^{(\sigma)}$ can be taken "close enough" to $\Lambda^{(0)}$, we decrease the size of the hole, i.e., we let $h \rightarrow 0$. Now 
the constructions of $\left(\Lambda^{(0)}, R^{(0)}\right)$ and $\left(\Lambda^{(\sigma)}, R^{(\sigma)}\right)$ are essentially identical. To avoid repeating ourselves more than needed, we will carry out the two constructions simultaneously. It is useful to keep in mind, however, that logically, the case of the infinitesimal hole is treated first, and some of the information so obtained is used to guide the arguments for positive-size holes.

As explained in Sect. 3.1, to ensure that the horseshoe respects the hole, it is convenient to include $f^{-1}\left(\partial H_{\sigma}\right)$ as part of the discontinuity set for $f$. Since $H_{\sigma}$ will be viewed as a perturbation of $H_{0}$, we include $f^{-1}\left(H_{0}\right)$ in this set as well. The following convention will be adopted when we consider a system with hole $H_{\sigma}$ :

(a) Suppose for definiteness $q_{0} \in \Gamma_{1}$. The new phase space $M_{\sigma}$ is obtained from $M$ by cutting $\Gamma_{1} \times\left[-\frac{\pi}{2}, \frac{\pi}{2}\right]$ along the lines comprising $H_{0} \cup \partial H_{\sigma}$, splitting it into three connected components.

(b) As a consequence, the new discontinuity set of $f$ is $f^{-1}\left(\partial M_{\sigma}\right)$, and the new discontinuity set of $f^{-1}$ is $f\left(\partial M_{\sigma}\right)$.

We use the notation " $\sigma=0$ " for the infinitesimal hole, so that $M_{0}$ is obtained from $M$ by cutting along $H_{0}$.

Notice immediately that this changes the definitions of stable and unstable curves, in the sense that if $\gamma$ was a stable curve for the system without holes, then $\gamma$ continues to be a stable curve if and only if (i) $\gamma \cap \partial M_{\sigma}=\emptyset$, and (ii) $f^{n}(\gamma) \cap f^{-1} \partial M_{\sigma}=\emptyset$ for all $n \geq 0$; a similar characterization holds for unstable curves. All objects constructed below will be $\sigma$-dependent, but we will suppress mention of $\sigma$ except where it is necessary. Observe also that the Important Geometric Facts $(\dagger)$ in Sect. 2.3 with $f^{-1} \partial M_{\sigma}$ instead of $f^{-1} \partial M$ as the new discontinuity set remains valid.

We now follow sequentially the 7 points outlined in Sect. 2.3 and discuss the modifications needed. These modifications, along with two additional points ( 8 and 9 ) form a complete proof of Proposition 2.2. We believe we have prepared ourselves adequately in Sects. 2.3 and 2.4 so that the discussion to follow can be understood on its own, but encourage readers who wish to see proofs complete with all technical detail to read the rest of this section alongside the relevant parts of $[\mathrm{Y}]$ and $[\mathrm{BSC} 2]$.

The notation within each item below is as in Sect. 2.3.

1. The relationships $\lambda=\lambda_{1}^{4}$ and $\delta=\delta_{1}^{4}$ are as before, and the sets $B_{\lambda_{1}, \delta_{1}}^{(\sigma) \pm}$ are defined in a manner similar to that in Sect. 2.3. For example,

$$
B_{\lambda_{1}, \delta_{1}}^{(\sigma)+}=\left\{x \in M_{\sigma}: d\left(x, \partial M_{\sigma}\right) \geq \delta_{1} \text { and } d\left(f^{n} x, f^{-1} \partial M_{\sigma}\right) \geq \delta_{1} \lambda_{1}^{-n} \forall n \geq 0\right\} .
$$

As in Sect. 2.3, the condition on $d\left(f^{n} x, f^{-1} \partial M_{\sigma}\right)$ is to ensure the existence of stable curves, and the necessity for $x$ to be away from $\partial M_{\sigma}$ is obvious (cf. footnote in item 1 of Section 2.3). Properties (i) and (ii) continue to hold for each $\sigma$ given the geometry of the new discontinuity set. With regard to the choice of $\delta_{1}$, we let $\delta_{1}$ be as in $[\mathrm{Y}]$, and shrink it if necessary to ensure that $B_{\lambda_{1}, 2 \delta_{1}}^{(0)+} \cap B_{\lambda_{1}, 2 \delta_{1}}^{(0)-}$ has positive $\nu$-measure away from $f^{-1}\left(\partial M_{0}\right) \cup \partial M_{0} \cup f\left(\partial M_{0}\right)$. This is where the sets $\Lambda^{(\sigma)}$ will be located (see Paragraph 2).

The following lemma relates $B_{\lambda_{1}, \delta_{1}}^{(\sigma) \pm}$ and $B_{\lambda_{1}, \delta_{1}}^{(0) \pm}$ : 
Lemma 3.2. (i) For all $\sigma \in \Sigma_{h}$, we have $B_{\lambda_{1}, \delta_{1}}^{(\sigma) \pm} \subset B_{\lambda_{1}, \delta_{1}}^{(0) \pm}$.

(ii) As $h \rightarrow 0$,

$$
\sup _{\sigma \in \Sigma_{h}} \nu\left(B_{\lambda_{1}, \delta_{1}}^{(0)+} \backslash B_{\lambda_{1}, \delta_{1}}^{(\sigma)+}\right) \rightarrow 0, \quad \sup _{\sigma \in \Sigma_{h}} \nu\left(B_{\lambda_{1}, \delta_{1}}^{(0)-} \backslash B_{\lambda_{1}, \delta_{1}}^{(\sigma)-}\right) \rightarrow 0 .
$$

Proof: (i) follows immediately from $\partial M_{\sigma} \supset \partial M_{0}$. As for (ii), let $\varepsilon>0$ be given. Recall that $N_{\alpha}(\cdot)$ denotes the $\alpha$-neighborhood of a set. By Lemma 3.1 we may choose $h$ small enough that for all $\sigma \in \Sigma_{h}, \partial H_{\sigma} \in N_{\varepsilon}\left(H_{0}\right)$ and $f^{-1}\left(\partial H_{\sigma}\right) \in N_{\varepsilon}\left(f^{-1} H_{0}\right)$. Then if $x \in B_{\lambda_{1}, \delta_{1}}^{(0)+} \backslash B_{\lambda_{1}, \delta_{1}}^{(\sigma)+}$, either $x \in N_{\delta_{1}}\left(\partial H_{\sigma}\right) \backslash N_{\delta_{1}}\left(H_{0}\right)$, or

$$
x \in \cup_{n \geq 0} f^{-n}\left(N_{\delta_{1} \lambda_{1}^{-n}}\left(f^{-1} \partial H_{\sigma}\right) \backslash N_{\delta_{1} \lambda_{1}^{-n}}\left(f^{-1} H_{0}\right)\right) .
$$

We estimate the $\nu$-measure of the right side separately for $\cup_{n \geq n_{\varepsilon}}$ and $\cup_{n<n_{\varepsilon}}$ where $n_{\varepsilon}=$ $\inf \left\{n \geq 0: \delta_{1} \lambda_{1}^{-n} \leq \varepsilon\right\} \approx \frac{\ln \left(\frac{\delta_{1}}{\varepsilon}\right)}{\ln \lambda_{1}}$. For $n \geq n_{\varepsilon}$, the measure in question is

$$
\leq \sum_{n \geq n_{\varepsilon}} \nu\left(N_{\delta_{1} \lambda_{1}^{-n}}\left(f^{-1}\left(\partial H_{\sigma}\right)\right) \leq \text { const } \cdot \sum_{n \geq n_{\varepsilon}} \delta_{1} \lambda_{1}^{-n} \leq \text { const } \cdot \varepsilon\right.
$$

Here we have used that $f^{-1}\left(\partial H_{\sigma}\right)$ consists of a finite number of smooth compact curves the total length of which is bounded independent of $\sigma$. Adding to this that these curves are within a distance $\varepsilon$ of the curves in $f^{-1} H_{0}$, we see that for each $n<n_{\varepsilon}$,

$$
\nu\left(N_{\delta_{1} \lambda_{1}^{-n}}\left(f^{-1}\left(\partial H_{\sigma}\right)\right) \backslash N_{\delta_{1} \lambda_{1}^{-n}}\left(f^{-1} H_{0}\right)\right) \leq \mathrm{const} \cdot \varepsilon .
$$

Similarly, $\nu\left(N_{\delta_{1}}\left(\partial H_{\sigma}\right) \backslash N_{\delta_{1}}\left(H_{0}\right)\right) \leq$ const $\cdot \varepsilon$. Hence

$$
\nu\left(B_{\lambda_{1}, \delta_{1}}^{(0)+} \backslash B_{\lambda_{1}, \delta_{1}}^{(\sigma)+}\right) \leq \mathrm{const} \cdot\left(\varepsilon+\left(n_{\varepsilon}+1\right) \varepsilon\right) \leq \mathrm{const} \cdot \varepsilon \ln \left(\frac{\delta_{1}}{\varepsilon}\right)
$$

which tends to 0 as $\varepsilon \rightarrow 0$.

2. To construct the Cantor sets, we first pick $x_{1}^{(0)}$ as a density point of $B_{\lambda_{1}, 2 \delta_{1}}^{(0)+} \cap B_{\lambda_{1}, 2 \delta_{1}}^{(0)-}$ at least $2 \delta_{1}$ away from $f^{-1}\left(\partial M_{0}\right) \cup \partial M_{0} \cup f\left(\partial M_{0}\right)$ and the boundaries of the homogeneity strips, and begin to construct $\Lambda^{(0)}$ with $\Omega=W_{\delta}^{u}\left(x_{1}^{(0)}\right)$. We then do the same for each $\sigma$, i.e., pick $x_{1}^{(\sigma)}$ as a density point of $B_{\lambda_{1}, 2 \delta_{1}}^{(\sigma)+} \cap B_{\lambda_{1}, 2 \delta_{1}}^{(\sigma)}$ and begin to construct $\Lambda^{(\sigma)}$ centered at $x_{1}^{(\sigma)}$ - except that for reasons to become clear, we will want $d\left(x_{1}^{(\sigma)}, x_{1}^{(0)}\right)<\delta_{2}$ where $\delta_{2}>0$ is determined by properties of $\left(\Lambda^{(0)}, R^{(0)}\right.$ ) (requirements will appear below, and in items 6 and $9)$. Suffice it to say here that however small $\delta_{2}$ may be, Lemma 3.2 guarantees that this can be done by shrinking $h$. Once $x_{1}^{(\sigma)}$ is chosen, we set $\Omega=W_{\delta}^{u}\left(x_{1}^{(\sigma)}\right)$ and

$$
\Omega_{n}=\left\{y \in \Omega: d\left(f^{i} y, f^{-1}\left(\partial M_{\sigma}\right)\right) \geq \delta_{1} \lambda_{1}^{-i} \text { for } 0 \leq i \leq n\right\} .
$$

Then the sets $\Omega_{\infty}, \Gamma^{s}, \Gamma^{u}$ and $\Lambda^{(\sigma)}$ are constructed as before.

That $Q\left(\Lambda^{(\sigma)}\right) \approx Q\left(\Lambda^{(0)}\right)$ follows immediately from the proximity of $x_{1}^{(\sigma)}$ to $x_{1}^{(0)}$. Since $\delta$ is fixed, $\mu^{u}\left(\Lambda^{(\sigma)}\right) \approx \mu^{u}\left(\Lambda^{(0)}\right)>0$ can be arranged by taking $\delta_{2}$ sufficiently small and using Lemma 3.2 with $h$ sufficiently small. This proves Proposition 2.2(b)(i). With the separation 
time happening sooner due to the enlarged discontinuity set, (P3)-(P5) remain true with the same $C$ and $\alpha$ for the closed system; in other words, Proposition 2.2(b)(iii) requires no further work.

3. To arrange for mixing properties (not done in $[Y]$ ), we will need to delay the return times to $\Lambda$ by forbidding returns before time $R_{2}$ for some $R_{2} \geq R_{1}$ determined by $\left(\Lambda^{(0)}, R^{(0)}\right)$; see Lemma 3.4. This aside, the construction of $f^{R}$ is as before. The matching of Cantor sets argument should be looked at again since the Cantor sets are different, but the proof goes through as before because the sets are dynamically defined.

Notice that for $\omega \in \tilde{\mathcal{P}}_{n}, f^{i} \omega$ is either entirely in the hole or outside of the hole, as is $f^{i}\left(\Lambda^{s}\right)$ where $\Lambda^{s}$ is the $s$-subset of $\Lambda$ associated with $\omega$, for $0 \leq i \leq n$; this is a direct consequence of our taking the boundary of the hole into consideration in our definition of the discontinuity set. Together with the fact that $\Lambda$ is away from $\partial M_{\sigma}$, it ensures that the generalized horseshoe we are constructing respects the hole.

4. This is where one of the more substantial modifications occur: Lemma 2.3, which is based largely on the competition between expansion along $u$-curves and the rate at which they are cut, is clearly affected by the additional cutting due to our enlarged discontinuity set. The condition $(*)$ in Sect. 2.3 must now be replaced by

Lemma 3.3. There exists $K_{1}$ such that for any $m \in \mathbb{Z}^{+}$, there exists $\varepsilon_{0}>0$ with the property that for any u-curve with $p(\omega)<\varepsilon_{0}, f^{m}(\omega)$ has $\leq\left(K_{1} m^{2}+4\right)$ connected components with respect to the enlarged discontinuity set.

Proof: Let $m \in \mathbb{Z}^{+}$be given. As in Sect. 2.3, choose $\varepsilon_{0}>0$ small enough such that if $\omega$ is a $u$-curve with $p(\omega)<\varepsilon_{0}, f^{m}(\omega)$ has $\leq\left(K_{0} m+1\right)$ connected components with respect to the original discontinuity set $f^{-1} S_{0}$. Let $\omega_{j}$ be the $f^{-m}$-image of one these connected components. This means that for $0 \leq k \leq m, f^{k}\left(\omega_{j}\right)$ is, in reality, a connected $u$-curve even though it may not be connected with respect to our enlarged discontinuity set. Since $f^{k} \omega_{j}$ is an (increasing) $u$-curve, it can meet the three vertical lines making up $\left(\partial H_{\sigma}\right) \cup H_{0}$ in no more than three points. (As the slopes $d \varphi / d r$ of $u$-curves are never less than the curvature of $\Gamma_{i}$ at $r$, connected $u$-curves cannot wrap around the cylinder $\Gamma_{i} \times[-\pi / 2, \pi / 2]$ and meet $\left(\partial H_{\sigma}\right) \cup H_{0}$ more than once.) Hence the cardinality of $\left\{\omega_{j} \cap \bigcup_{k=0}^{m} f^{-k}\left(\left(\partial H_{\sigma}\right) \cup H_{0}\right)\right\}$ is $\leq 3(m+1)$, and as $\left(\partial H_{\sigma}\right) \cup H_{0}$ is the additional set added to $\partial M$ to create $\partial M_{\sigma}$, it follows that $f^{m} \omega$ has $\leq\left(K_{0} m+1\right) \cdot(3(m+1)+1)$ connected components with respect to the enlarged discontinuity set.

Using Lemma 3.3, one adapts easily the proof of Lemma 2.3 to the present setup with $\theta_{1}=\left(K_{1} m^{2}+4\right)^{\frac{1}{m}}\left(\lambda^{-1}+\alpha_{0}\right)$, where $m$ is chosen large enough so that this number is $<1$. The constant $D_{1}$ depends only on the properties of $D f$ and is unchanged. Hence Lemma 2.3 is valid with $D_{1}$ and $\theta_{1}$ modified but independent of $\sigma$. As in Section 2.3, these estimates can then be adapted to estimate $p\left(\omega_{n} \backslash\{T \leq n\}\right)$.

5. Lemma 2.4 remains valid with modified constants which are independent of $\sigma$. Returning to the sketch of the proof provided in Sect. 2.3, we see that both sets of estimates boil down to the geometry of the new discontinuity set and the rates of growth versus cutting, which has been taken care of for the enlarged discontinuity set in Paragraph 4 above. 
6. We need to show that there exist $n_{1}$ and $\varepsilon_{1}>0$ independent of $\sigma$ such that for every homogeneous $u$-curve with $p$-length $>\varepsilon_{0}$, a fraction $\geq \varepsilon_{1}$ of $\omega$ returns within the next $n_{1}$ steps. Before we enlarged the discontinuity set, this property followed from property $\left({ }^{* *}\right)$ in Sect. 2.3. We replace $(* *)$ here with the following:

Lemma 3.4. Given $\varepsilon_{0}>0$, provided $h$ and $\delta_{2}$ are sufficiently small, there exists $n_{1}$ such that the following holds for each $\sigma \in \Sigma_{h}$ : for every homogeneous $W_{\text {loc }}^{u}$-curve $\omega$ with $p(\omega)>\varepsilon_{0}$ and each $q \in\left\{n_{1}, n_{1}+1\right\}, f^{q} \omega$ contains a homogeneous segment that $u$-crosses the middle half of $Q\left(\Lambda^{(\sigma)}\right)$ with greater than $2 \delta$ sticking out from each side.

Once Lemma 3.4 is proved, the fact that a fraction $\varepsilon_{1}$ (independent of $\sigma$ ) has the desired properties follows from derivative estimates as in Sect. 2.3 and our uniform lower bound on $\mu^{u}\left(\Lambda^{(\sigma)}\right)$. The reason we want $q$ to take two consecutive values in the statement of Lemma 3.4 has to do with the mixing property in item 9 below.

Proof: Fix $\varepsilon_{0}>0$. We first prove the following for the case $\sigma=0$ :

(**)' For $\sigma=0$, there exists $n_{1}$ such that any homogeneous $W_{\text {loc }}^{u}$-curve $\omega$ with $p(\omega)>\varepsilon_{0}$ and every $q \geq n_{1}, f^{q} \omega$ contains a homogeneous segment that $u$-crosses the middle fourth of $Q\left(\Lambda^{(0)}\right)$ with greater than $4 \delta$ sticking out from each side.

The proof of $\left({ }^{* *}\right)^{\prime}$ is completely analogous to the proof of $\left(^{* *}\right)$ outlined in Sect. 2.4. Sublemmas A and B continue to hold due to the similar geometry of the discontinuity set.

Notice that unlike (**)', the assertion in Lemma 3.4 is only for $q=n_{1}$ and $n_{1}+1$, so that its proof involves only a finite number of mixing boxes $U_{j}$ and a finite number of iterates. This will be important in the perturbative argument to follow.

Consider now $\sigma \neq 0$, and consider a homogeneous unstable curve $\omega$ with $p(\omega)>\varepsilon_{0}$. First, $\omega$ continues to be an unstable curve with respect to the discontinuity set $f^{-1} \partial M_{0}$, so by the proof of $\left({ }^{* *}\right)^{\prime}$, for $q \in\left\{n_{1}, n_{1}+1\right\}$ and every $j$, there is a rectangular region $Q^{*}=Q^{*}(q, j)$ such that (i) $Q^{*} u$-crosses the middle fourth of $Q\left(\Lambda^{(0)}\right)$ with $>4 \delta$ sticking out, (ii) $f^{-q} Q^{*}$ is an $s$-subrectangle in the middle third of $Q\left(U_{j}\right)$, and (iii) for $i=0,1, \cdots, q, f^{-i} Q^{*}$ stays clear of $f^{-1} \partial M_{0}$ by some amount. Lemma 3.1 ensures that for $h$ small enough, (iii) continues to hold with $f^{-1} \partial M_{0}$ replaced by $f^{-1} \partial M_{\sigma}$. Finally, provided $\delta_{2}$ is small enough, (i) holds for $Q\left(\Lambda^{(\sigma)}\right)$ with $>2 \delta$ sticking out on each side.

7. Once steps 4,5 and 6 have been completed, the argument here is unchanged (as it is largely combinatorial), guaranteeing constants $C_{0}$ and $\theta_{0}$ independent of $\sigma$ with $p\{R \geq n\} \leq C_{0} \theta_{0}^{n}$. This completes the proof of Proposition 2.2(a)(i) and (b)(ii).

We have reached the end of the 7 steps outlined in Sect. 2.3. Two items remain:

8. That $\bar{n}(h) \rightarrow \infty$ as $h \rightarrow 0$ is easy: Orbits from $\Lambda^{(\sigma)}$ start away from $H_{0}$ and cannot approach $f^{-1} H_{0}$ faster than a fixed rate. Thus using Lemma 3.1, we can arrange for orbits starting from $\Lambda^{(\sigma)}$ to stay out of $H_{\sigma}$ for as long as we wish by taking $h$ small.

9. The mixing of $\left(\Lambda^{(\sigma)}, R^{(\sigma)}\right)$ follows from 
Lemma 3.5. There exists $R_{2} \geq R_{1}$ (independent of $\sigma$ ) such that for small enough $h$, the construction in Step 3 can be modified to give the following:

(i) no returns are allowed before time $R_{2}$, and

(ii) at both times $R_{2}$ and $R_{2}+1$, there are s-subsets of $\Lambda^{(\sigma)}$ making full returns.

Proof: Again we first consider the case $\sigma=0$. Here $R_{2}$ is chosen as follows: Without allowing any returns, let $R_{1}^{\prime}$ be the smallest time greater than or equal to $R_{1}$ such that there exists $\omega \in \tilde{\mathcal{P}}_{R_{1}^{\prime}}$ with $p\left(f^{R_{1}^{\prime}} \omega\right)>\varepsilon_{0}>0$. With $\varepsilon_{0}$ chosen as before, we take $n_{1}$ from Lemma 3.4 and set $R_{2}=R_{1}^{\prime}+n_{1}$. Using Lemma 3.4, we find two subsegments $\omega^{\prime}$ and $\omega^{\prime \prime} \subset \omega$ such that $f^{R_{2}} \omega^{\prime}$ and $f^{R_{2}+1} \omega^{\prime \prime}$ are both homogeneous segments that $u$-cross the middle half of $Q\left(\Lambda^{(0)}\right)$ with greater than $2 \delta$ sticking out from each side. We may suppose that $\omega^{\prime}$ and $\omega^{\prime \prime}$ are disjoint since $f$ has no fixed points. They give rise to two $s$-subsets of $\Lambda^{(0)}$ with the properties in (ii). From time $R_{2}$ on, returns to $\Lambda^{(0)}$ are allowed as before.

When $\sigma \neq 0$, we follow the same procedure as above to ensure the mixing of $\left(\Lambda^{(\sigma)}, R^{(\sigma)}\right)$. The only concern is that $R_{1}^{\prime}=R_{1}^{\prime}(\sigma)$ (and hence also $R_{2}=R_{1}^{\prime}+n_{1}$ ) might not be independent of $\sigma$. This is not a problem as the construction above involves only a finite number of steps: With $h$ and $\delta_{2}$ sufficiently small, the elements of $\tilde{\mathcal{P}}_{n}^{(\sigma)}$ can be defined in such a way that they are in a one-to-one correspondence with those of $\tilde{\mathcal{P}}_{n}^{(0)}$ for $n \leq R_{1}^{\prime}(0)$.

Finally, mixing of the surviving dynamics is ensured by choosing $h$ small enough that $\bar{n}(h)>R_{2}+1$. This ensures that the $s$-subsets $\Lambda^{s}$ that make full returns at times $R_{2}$ and $R_{2}+1$ cannot fall into the hole prior to returning.

The proof of Proposition 2.2 for holes of Type I is now complete.

\subsection{Modifications needed for holes of Type II}

The proof for Type II holes is very similar to that for Type I holes. There are, however, some differences due to the more complicated geometry of $\partial H_{\sigma}$. In the discussion below, we assume the infinitesimal hole $\left\{q_{0}\right\}$ does not lie on any segment in the table tangent to more than one scatterer. The general situation is left to the reader.

From the discussion of the geometry of Type II holes in Sect. 3.1, we see that the Important Geometric Facts $(\dagger)$ in Sect. 2.3 continue to hold with $M_{\sigma}$ in the place of $M$, except that $u$-curves need not be transversal to the $\partial H_{\sigma} \cup H_{0}$ part of $\partial M_{\sigma}$. Potential problems that may arise are discussed below. The discontinuity set of $f$, i.e. $f^{-1} \partial M_{\sigma}$, has the same geometric properties as before.

We now go through the 9 points in Sect. 3.2. No modifications are needed in items 1-3. As expected, item 4 is where the most substantial modifications occur:

Modifications in Item 4. Lemma 3.3 is still true as stated, but the geometry is different. In the discussion below related to this lemma, the discontinuity set refers to $f^{-1} \partial M$, not the enlarged discontinuity set $f^{-1} \partial M_{\sigma}$, and unstable curves are defined accordingly. For Type I holes, the proof relies on the fact that any (increasing) connected $u$-curve $\omega$ meets $\partial H_{\sigma} \cup H_{0}$, which is the union of three vertical lines, in at most three points.

Lemma 3.6. Any unstable curve $\omega$ meets $\partial H_{\sigma} \cup H_{0}$ in at most three points. 
Even though Lemma 3.3 is stated for $u$-curves, we need it only for unstable curves (and the argument here is slightly simpler for unstable curves).

Proof. Let us distinguish between two different types of curves that comprise $\partial H_{\sigma}$ : Primary segments, which are the forward images of curves in $\partial B_{\sigma} \backslash f^{-1}(\partial M)$, and secondary segments, which are subsegments of $f(\partial M)$. For examples, see Fig. 4. In general, when $q_{0}$ does not lie on a line segment with multiple tangencies to the scatterers, secondary segments are absent in $H_{0}$, while each component of $H_{0}$ gives rise to two primary segments in $\partial H_{\sigma}$ for $\sigma \neq 0$.

To prove the lemma, observe first that $H_{0}$ can have no more than one component in any connected component of $M \backslash f(\partial M)$. Second, $\omega$ must also be entirely contained inside one connected component of $M \backslash f(\partial M)$. This is because unstable curves for $f$ cannot cross the discontinuity set of $f^{-1}$. As a consequence, $\omega$ also cannot cross any secondary segment as secondary segments of $\partial H_{\sigma}$ are contained in $f(\partial M)$.

It remains to show that $\omega$ can meet each primary segment in at most one point. Although primary segments are increasing, their tangent vectors lie outside of unstable cones (except at $\partial M$ where the unstable cone is degenerate). This is because the curves in $\partial B_{\sigma} \backslash f^{-1} \partial M$ are decreasing, while the unstable cones are defined to be the forward images of $\left\{0 \leq \frac{d \varphi}{d r} \leq \infty\right\}$ under $D f$. Hence primary segments have greater slopes than $\omega$.

As pointed out in Sect. 2.3, item 4, Lemma 3.3 must be modified to account for the deletions that arise from intersections with forward images of $\Omega_{n}$, and one might be concerned about the absence of uniform estimates on transversality in $(\dagger)$ between $\partial H_{\sigma}$ and unstable curves. This, in fact, is not a problem, because such deletions occur only in neighborhoods of $f^{-1} \partial M_{\sigma}$, which are decreasing curves and hence uniformly transversal to $u$-curves.

This completes the modifications associated with item 4.

No modifications are required for items 5, 7,8 and 9.

Modifications in Item 6. In the proof of $\left({ }^{* *}\right)^{\prime}$, the argument needs to be modified, again due to the difference in geometry: In order to prove that $Q^{*} \cap\left(\cup_{0}^{q} f^{i}\left(\partial M_{0}\right)\right)=\emptyset$ (Sublemma B), in the case of Type I holes we use that $\cup_{1}^{q} f^{i}\left(\partial M_{0}\right)$ is the union of finitely many piecewise smooth increasing curves that stretch from $\left\{\varphi=-\frac{\pi}{2}\right\}$ to $\left\{\varphi=\frac{\pi}{2}\right\}$. For Type II holes, this is not true. However, it can be arranged that Sublemma B will continue to hold as we now explain: First,

$$
\cup_{1}^{q} f^{i}\left(\partial M_{0}\right) \subset\left(\left(\cup_{1}^{q} f^{i}(\partial M)\right) \cup\left(\cup_{0}^{q-1} f^{i}\left(H_{0}\right)\right)\right) \cup f^{q}\left(H_{0}\right)
$$

If we write the right side as $A \cup f^{q}\left(H_{0}\right)$, then $A$ has the desired geometry, i.e. it is the union of finitely many piecewise smooth increasing curves that stretch from $\left\{\varphi=-\frac{\pi}{2}\right\}$ to $\left\{\varphi=\frac{\pi}{2}\right\}$. Thus the same argument as before shows that this set is disjoint from $Q^{*}$. One way to ensure that $Q^{*} \cap f^{q}\left(H_{0}\right)=\emptyset$ is to choose the mixing boxes $U_{j}$ disjoint from $H_{0}$, which can easily be arranged given the geometry of primary segments discussed above.

This completes the proof of Proposition 2.2 for Type II holes. 

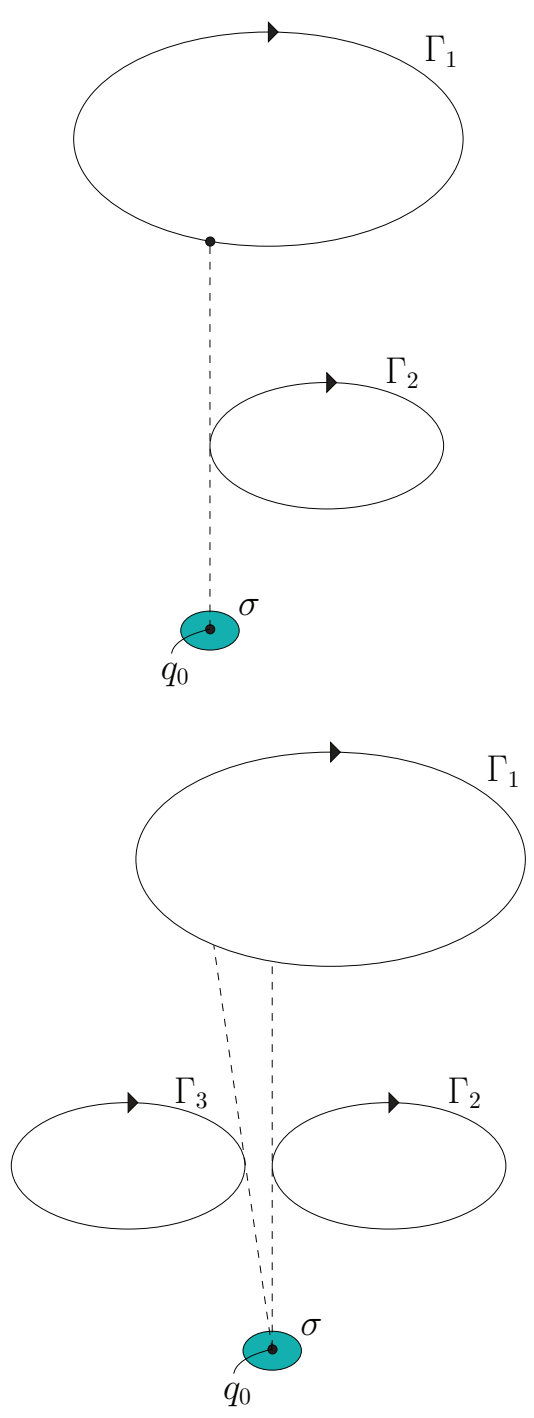
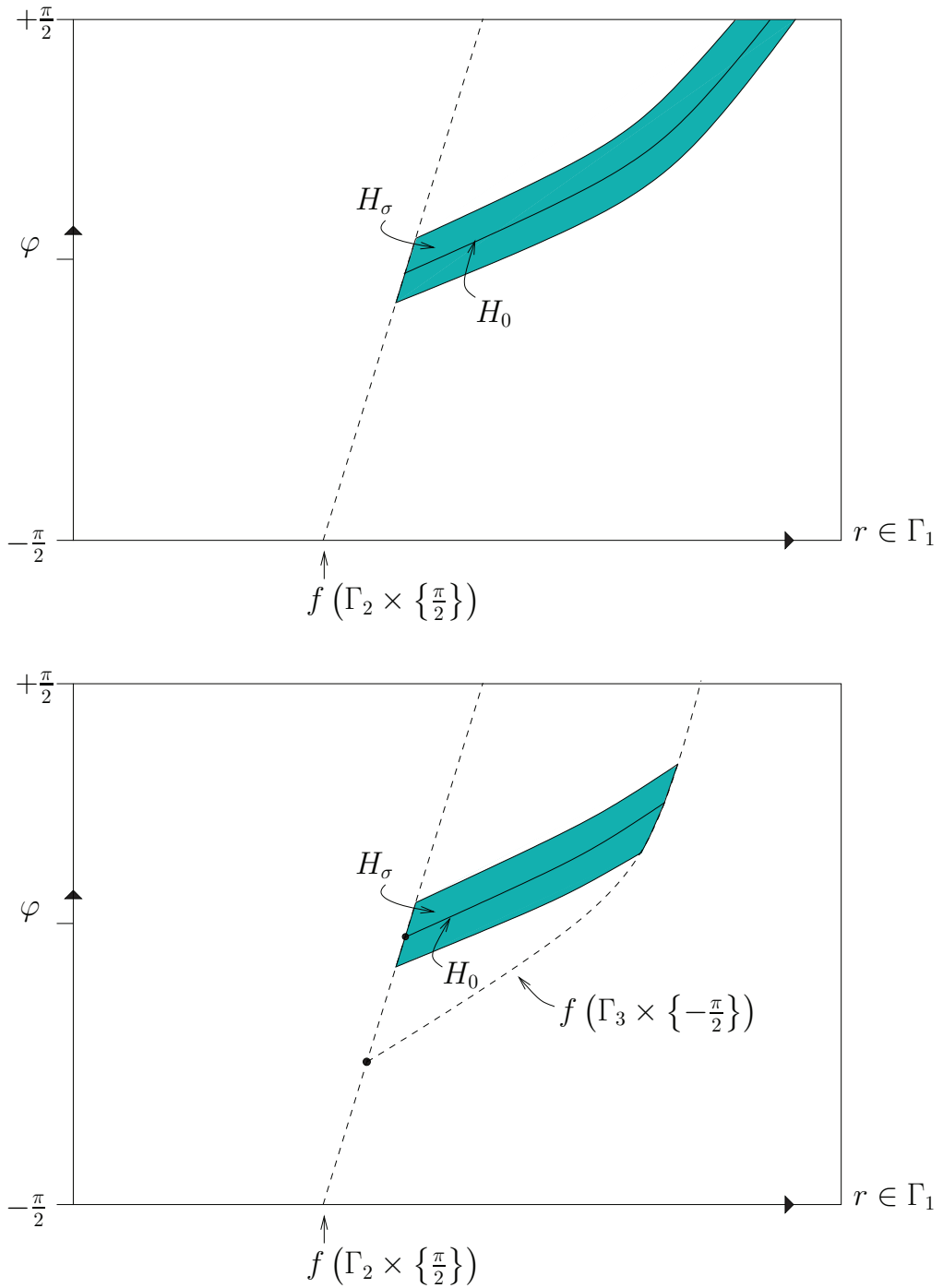

Figure 4: Representative examples of the geometry of Type II holes. Top set: On the left is a configuration on the billiard table $X$, while on the right is the resulting configuration in the subset $\Gamma_{1} \times\left[-\frac{\pi}{2}, \frac{\pi}{2}\right]$ of phase space. In this subset, $H_{0}$ consists of a single primary segment whose endpoints lie on $f\left(\Gamma_{2} \times\left\{\frac{\pi}{2}\right\}\right)$ and $\Gamma_{1} \times\left\{\frac{\pi}{2}\right\}$. For $\sigma \neq 0, \partial H_{\sigma}$ contains two primary segments and a single secondary segment that lies on $f\left(\Gamma_{2} \times\left\{\frac{\pi}{2}\right\}\right)$. (Recall that by convention $\partial H_{\sigma}$ does not include subsegments of $\partial M$.) Bottom set: The analogous situation when the view of $\Gamma_{1}$ from $q_{0}$ is obstructed by two scatterers, instead of just one. Observe that now $\partial H_{\sigma}$ contains two secondary segments in $\Gamma_{1} \times\left[-\frac{\pi}{2}, \frac{\pi}{2}\right]$. The situation when the view of $\Gamma_{1}$ from $q_{0}$ is unobstructed by other scatterers is simple and is left to the reader. 


\section{Escape Dynamics on Markov Towers}

In this section and the next, we lift the problems from the billiard systems in question to their Markov tower extensions, and solve the problems there. In Sect. 4, we review relevant works and formulate results on towers. Proofs are given in Sect. 5.

\subsection{From generalized horseshoes to Markov towers (review)}

It is shown in $[\mathrm{Y}]$ that given a map $f: M \rightarrow M$ with a generalized horseshoe $(\Lambda, R)$ as defined in Sect. 2.1, one can associate a Markov extension $F: \Delta \rightarrow \Delta$ which focuses on the return dynamics to $\Lambda$ (and suppresses details between returns). We first recall some facts about this very general construction, taking the opportunity to introduce some notation.

Let

$$
\Delta=\{(x, n) \in \Lambda \times \mathbb{N}: n<R(x)\},
$$

and define $F: \Delta \rightarrow \Delta$ as follows: For $\ell<R(x)-1$, we let $F(x, \ell)=(x, \ell+1)$, and define $F(x, R(x)-1)=\left(f^{R(x)}(x), 0\right)$. Equivalently, one can view $\Delta$ as the disjoint union $\cup_{\ell \geq 0} \Delta_{\ell}$ where $\Delta_{\ell}$, the $\ell^{\text {th }}$ level of the tower, is a copy of $\{x \in \Lambda: R(x)>\ell\}$. This is the representation we will use. There is a natural projection $\pi: \Delta \rightarrow M$ such that $\pi \circ F=f \circ \pi$. In general, $\pi$ is not one-to-one, but for each $\ell \geq 0$, it maps $\Delta_{\ell}$ bijectively onto $f^{\ell}(\Lambda \cap\{R \geq \ell\})$.

In the construction of $(\Lambda, R)$, one usually introduces an increasing sequence of partitions of $\Lambda$ into $s$-subsets representing distinguishable itineraries in the first $n$ steps. (In Sects. 2.3 and 3.2 , these partitions were given by $\tilde{\mathcal{P}}_{\ell}$ of $\tilde{\Omega}_{\ell}$.) These partitions induce a partition $\left\{\Delta_{\ell, j}\right\}$ of $\Delta$ which is finite on each level $\ell$ and and is a (countable) Markov partition for $F$. We define a separation time $s(x, y) \leq s_{0}(x, y)$ by $\inf \left\{n>0: F^{n} x, F^{n} y\right.$ lie in different $\left.\Delta_{\ell, j}\right\}$.

We borrow the following language from $(\Lambda, R)$ for use on $\Delta$ : For each $\ell, j$, recall that $\Gamma^{s}\left(\pi\left(\Delta_{\ell, j}\right)\right)$ and $\Gamma^{u}\left(\pi\left(\Delta_{\ell, j}\right)\right)$ are the stable and unstable families defining the hyperbolic product set $\pi\left(\Delta_{\ell, j}\right)$. We will say $\tilde{\gamma} \subset \Delta_{\ell, j}$ is an unstable leaf of $\Delta_{\ell, j}$ if $\pi(\tilde{\gamma})=\gamma \cap \pi\left(\Delta_{\ell, j}\right)$ for some $\gamma \in \Gamma^{u}\left(\pi\left(\Delta_{\ell, j}\right)\right)$, and use $\Gamma^{u}\left(\Delta_{\ell, j}\right)$ to denote the set of all such $\tilde{\gamma}$. Let $\Gamma^{u}(\Delta)=$ $\cup_{\ell, j} \Gamma^{u}\left(\Delta_{\ell, j}\right)$ be the set of all unstable leaves of $\Delta$. Stable leaves of $\Delta_{\ell, j}$ and the families $\Gamma^{s}\left(\Delta_{\ell, j}\right)$ and $\Gamma^{s}(\Delta)$ are defined similarly.

Associated with $F: \Delta \rightarrow \Delta$, which we may think of as a "hyperbolic tower", is its quotient "expanding tower" obtained by collapsing stable leaves to points. Topologically, $\bar{\Delta}=\Delta / \sim$ where for $x, y \in \Delta, x \sim y$ if and only if $y \in \gamma(x)$ for some $\gamma \in \Gamma^{s}(\Delta)$. Let $\bar{\pi}: \Delta \rightarrow \bar{\Delta}$ be the projection defined by $\sim$, and let $\bar{F}: \bar{\Delta} \rightarrow \bar{\Delta}$ be the induced map on $\bar{\Delta}$ satisfying $\bar{F} \circ \bar{\pi}=\bar{\pi} \circ F$. We will use the notation $\bar{\Delta}_{\ell}=\bar{\pi}\left(\Delta_{\ell}\right), \bar{\Delta}_{\ell, j}=\bar{\pi}\left(\Delta_{\ell, j}\right)$, and so on.

It is shown in $[\mathrm{Y}]$ that there is a well defined differential structure on $\bar{\Delta}$ preserved by $\bar{F}$. Recall that $\mu_{\gamma}$ is the Riemannian measure on $\gamma$, and for $\gamma, \gamma^{\prime} \in \Gamma^{u}(\Lambda), \Theta_{\gamma, \gamma^{\prime}}: \gamma \cap \Lambda \rightarrow \gamma^{\prime} \cap \Lambda$ is the holonomy map obtained by sliding along stable curves, i.e. $\Theta_{\gamma, \gamma^{\prime}}(x)=\gamma^{s}(x) \cap \gamma^{\prime}$. We introduce the following notation: For $x \in \Lambda_{i} \cap \gamma$, let $\gamma^{\prime}$ be such that $f^{R_{i}}(\gamma) \subset \gamma^{\prime}$. Then $J^{u}\left(f^{R}\right)(x)=J_{m_{\gamma}, m_{\gamma^{\prime}}}\left(f^{R_{i}} \mid\left(\gamma \cap \Lambda_{i}\right)\right)(x)$ is the Jacobian of $f^{R}$ with respect to the measures $m_{\gamma}$ and $m_{\gamma^{\prime}}$. Lemma 1 of [Y], which we recall below, is key to the differential structure on $\bar{\Delta}$.

Lemma 4.1. There is a function $u: \Lambda \rightarrow \mathbb{R}$ such that for each $\gamma \in \Gamma^{u}(\Lambda)$, if $m_{\gamma}$ is the measure whose density with respect to $\mu_{\gamma}$ is $e^{u} I_{\gamma \cap \Lambda}$, then we have the following: 
(1) For all $\gamma, \gamma^{\prime} \in \Gamma^{u}(\Lambda),\left(\Theta_{\gamma, \gamma^{\prime}}\right)_{*} m_{\gamma}=m_{\gamma^{\prime}}$.

(2) $J^{u}\left(f^{R}\right)(x)=J^{u}\left(f^{R}\right)(y)$ for all $y \in \gamma^{s}(x)$.

(3) $\exists C_{1}>0$ (depending on $C$ and $\alpha$ ) such that for each $i$ and all $x, y \in \Lambda_{i} \cap \gamma$,

$$
\left|\frac{J^{u}\left(f^{R}\right)(x)}{J^{u}\left(f^{R}\right)(y)}-1\right| \leq C_{1} \alpha^{s\left(f^{R} x, f^{R} y\right) / 2} .
$$

The properties of $u$ include $|u| \leq C$ and $|u(x)-u(y)| \leq 4 C \alpha^{\frac{1}{2} s(x, y)}$ on each $\gamma$.

(1) and (2) together imply that there is a natural measure $\bar{m}$ on $\bar{\Delta}$ with respect to which the Jacobian of $\bar{F}, J \bar{F}$, is well defined: First, identify $\bar{\Delta}_{0}$ with $\gamma \cap \Lambda$ for any $\gamma \in \Gamma^{u}(\Lambda)$, and let $\left.\bar{m}\right|_{\Delta_{0}}$ be the measure that corresponds to $m_{\gamma}$. (1) says that $\bar{m}$ so defined is independent of $\gamma$, and (2) says that with respect to $\bar{m}, J \bar{F}^{R}(x)=J^{u}\left(f^{R}\right)(y)$ for any $y \in \gamma^{s}(x)$. We then extend $\bar{m}$ to $\cup_{\ell>0} \bar{\Delta}_{\ell}$ in such a way that $J \bar{F} \equiv 1$ on all of $\bar{\Delta} \backslash \bar{F}^{-1}\left(\bar{\Delta}_{0}\right)$.

In the rest of Sect. 4.1 we will assume $\bar{m}\{R>n\}<C_{0} \theta_{0}^{n}$ for some $C_{0} \geq 1$ and $\theta_{0}<1$. $^{6}$

One of the reasons for passing from the hyperbolic tower to the expanding tower is that the spectral properties of the transfer operator associated with the latter can be leveraged. We fix $\beta$ with $1>\beta>\max \left\{\theta_{0}, \sqrt{\alpha}\right\}$, and define a symbolic metric on $\bar{\Delta}$ by $d_{\beta}(x, y)=\beta^{s(x, y)}$. Since $\beta>\sqrt{\alpha}$, Lemma 4.1(3) implies that $J \bar{F}$ is log-Lipshitz with respect to this metric. A natural function space on $\bar{\Delta}$ is $\mathcal{B}=\left\{\rho \in L^{1}(\bar{\Delta}, \bar{m}):\|\rho\|<\infty\right\}$ where $\|\rho\|=\|\rho\|_{\infty}+\|\rho\|_{\text {Lip }}$ and

$$
\|\rho\|_{\infty}=\sup _{\ell, j} \sup _{x \in \bar{\Delta}_{\ell, j}}|\rho(x)| \beta^{\ell}, \quad\|\rho\|_{\text {Lip }}=\sup _{\ell, j} \operatorname{Lip}\left(\left.\rho\right|_{\bar{\Delta}_{\ell, j}}\right) \beta^{\ell} .
$$

$\operatorname{Lip}(\cdot)$ above is with respect to the symbolic metric $d_{\beta}$. The weights $\beta^{\ell}$ provide the needed contraction from one level to the next, and $\beta>\theta_{0}$ is needed to maintain exponential tail estimates.

\subsection{Towers with Markov holes}

Now consider a leaky system $(f, M, H)$ as defined in Sect. 2.1, and suppose $(\Lambda, R)$ is a generalized horseshoe respecting the hole $H$. Let $F: \Delta \rightarrow \Delta$ be the associated tower map with $\pi: \Delta \rightarrow M$, and let $\tilde{H}=\pi^{-1}(H)$. Then $(F, \Delta, \tilde{H})$ is a leaky system in itself. With the horseshoe respecting $H$, we have that $\tilde{H}$ is the union of a collection of $\Delta_{\ell, j}$, usually an infinite number of them; we refer to holes of this type as "Markov holes". The notation $H_{\ell}:=\tilde{H} \cap \Delta_{\ell}$ will be used. Projecting and letting $\bar{H}=\bar{\pi}(\tilde{H})$, we obtain the quotient leaky system $(\bar{F}, \bar{\Delta}, \bar{H})$. Let us say $(F, \Delta, \tilde{H})$ and $(\bar{F}, \bar{\Delta}, \bar{H})$ are mixing if the surviving dynamics of the horseshoe that gives rise to these towers are mixing; see Sect. 2.1.

Letting $\stackrel{\Delta}{\Delta}=\Delta \backslash \tilde{H}$, we introduce the notation

$$
\Delta^{n}=\cap_{i=0}^{n} F^{-1} \stackrel{\triangleright}{\Delta}=\left\{x \in \Delta: F^{i} x \notin \tilde{H} \text { for } 0 \leq i \leq n\right\},
$$

so that in particular $\stackrel{\Delta}{=}=\Delta^{0}$. Corresponding objects for $(\bar{F}, \bar{\Delta}, \bar{H})$ are denoted by $\bar{\Delta}^{n}$.

\footnotetext{
${ }^{6}$ Our default rule is to use the same symbol for corresponding objects for $f, F$ and $\bar{F}$ when no ambiguity can arise given context. Thus $R$ is the name of the return time function on $\Lambda, \Delta_{0}$ and $\bar{\Delta}_{0}$.
} 


\subsubsection{What is known: Spectral properties of expanding towers}

Expanding towers (that are not necessarily quotients of hyperbolic towers) with Markov holes were studied in [D1] and [BDM]. The following theorem summarizes several results proved in [BDM, Proposition 2.4, Corollary 2.5], under some conditions on the tower that are easily satisfied here. We refer the reader to $[\mathrm{BDM}]$ for detail, and state their results in our context of $(\bar{F}, \bar{\Delta}, \bar{H})$.

Let $\stackrel{\circ}{\mathcal{B}}=\left\{\rho \in L^{1}\left(\bar{\Delta}^{0}, \bar{m}\right):\|\rho\|<\infty\right\}$ where $\|\rho\|$ is as above, and let $\overline{\mathcal{L}}$ denote the transfer operator associated with $\left.\bar{F}\right|_{\Delta^{1}}$ defined on $\dot{\mathcal{B}}$, i.e., for $\rho \in \dot{\mathcal{B}}$ and $x \in \bar{\Delta}^{0}$,

$$
\overline{\mathcal{L}} \rho(x)=\sum_{y \in \bar{\Delta}^{0} \cap \bar{F}^{-1} x} \rho(y)(J \bar{F}(y))^{-1} .
$$

Theorem 4.2. [BDM] Let $(\bar{F}, \bar{\Delta}, \bar{H})$ be such that $(i)(\bar{F}, \bar{\Delta})$ has exponential return times and (ii) $(\bar{F}, \bar{\Delta}, \bar{H})$ is mixing. Assume the following condition on hole size:

$$
\sum_{\ell \geq 1} \beta^{-(\ell-1)} \bar{m}\left(H_{\ell}\right)<\frac{(1-\beta) \bar{m}\left(\bar{\Delta}_{0}\right)}{1+C_{1}} .
$$

Then the following hold:

(1) $\overline{\mathcal{L}}$ is quasi-compact with a unique eigenvalue $\vartheta_{*}$ of maximum modulus; $\vartheta_{*}$ is real and

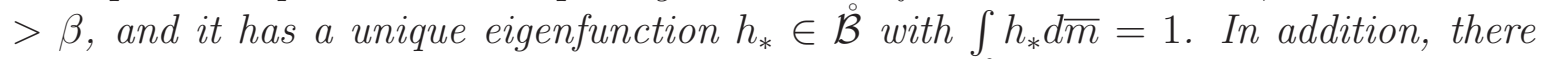
exist constants $D>0$ and $\tau<1$ such that for all $\rho \in \mathcal{B}$,

$$
\left\|\vartheta_{*}^{-n} \overline{\mathcal{L}}^{n} \rho-d(\rho) h_{*}\right\| \leq D\|\rho\| \tau^{n}, \quad \text { where } d(\rho)=\lim _{n \rightarrow \infty} \lambda^{-n} \int_{\bar{\Delta}^{n}} \rho d \bar{m}<\infty .
$$

(2) The eigenvalue $\vartheta_{*}$ satisfies $\vartheta_{*}>1-\frac{1+C_{1}}{\bar{m}\left(\bar{\Delta}_{0}\right)} \sum_{\ell \geq 1} \beta^{-(\ell-1)} \bar{m}\left(H_{\ell}\right)$.

The spectral property of $\overline{\mathcal{L}}$ as described in Theorem 4.2(1) implies that all $\rho$ except for those in a codimension 1 subspace have $d(\rho) \neq 0$. Given the pivotal role played by the base $\bar{\Delta}_{0}$ of the tower $\bar{\Delta}$, one would guess that for a density $\rho$, if $\rho>0$ on $\bar{\Delta}_{0}$, then $d(\rho) \neq 0$. A slightly more general condition is given in Corollary 4.3 below. We call $\bar{\Delta}_{\ell, j}$ a surviving element of the tower if some part of $\bar{\Delta}_{\ell, j}$ returns to $\bar{\Delta}_{0}$ before entering $\bar{H}$.

Corollary 4.3. [BDM] Let $\rho \in \dot{\mathcal{B}}$ be a nonnegative function that is $>0$ on a surviving $\bar{\Delta}_{\ell, j}$. Then $d(\rho)>0$.

\subsubsection{What is desired: Results for hyperbolic towers}

Here we formulate a set of results for the hyperbolic tower that connect the results in Sect. 4.2.1 to the stated theorems for billiards. Let $\tilde{\mathcal{G}}$ be the class of measures $\eta$ on $\Delta$ with the following properties: (i) $\eta$ has absolutely continuous conditional measures on unstable leaves; and (ii) $\bar{\pi}_{*} \eta=\bar{\rho} d \bar{m}$ for some $\bar{\rho} \in \dot{\mathcal{B}}$ with $d(\bar{\rho})>0$.

Let $\left(\Lambda^{(\sigma)}, R^{(\sigma)}\right)$ be a generalized horseshoe with the properties in Proposition 2.2, and let $(F, \Delta)$ be its associated tower. Let $n(\Delta, \tilde{H}):=\sup \left\{\ell: H_{\ell}=\emptyset\right\}$, i.e., $n(\Delta, \tilde{H})=$ $n\left(\Lambda^{(\sigma)}, R^{(\sigma)} ; H_{\sigma}\right)$ as defined in Sect. 2.2. 
Theorem 4.4. Assume that $n(\Delta, \tilde{H})$ is large enough that

$$
\sum_{\ell \geq n(\Delta, \tilde{H})} \beta^{-(\ell-1)} \bar{m}\left(\bar{\Delta}_{\ell}\right)<\frac{(1-\beta) \bar{m}\left(\bar{\Delta}_{0}\right)}{1+C_{1}}
$$

Then the following hold:

(a) There exists $\vartheta_{*}<1$ such that for all $\eta \in \tilde{\mathcal{G}}$,

$$
\log \vartheta_{*}=\lim _{n \rightarrow \infty} \frac{1}{n} \log \eta\left(\Delta^{n}\right)
$$

(b) There exists a conditionally invariant distribution $\tilde{\mu}_{*} \in \tilde{\mathcal{G}}$ with escape rate $-\log \vartheta_{*}$ for which the following hold: For all $\eta \in \tilde{\mathcal{G}}$, if $\bar{\rho}$ is the density of $\bar{\pi}_{*} \eta$ and $d(\bar{\rho})$ is as in Theorem 4.2, then

$$
\lim _{n \rightarrow \infty} \frac{\stackrel{\circ}{F}_{*}^{n} \eta}{\stackrel{\circ}{F}_{*}^{n} \eta(\stackrel{\circ}{\Delta})}=\tilde{\mu}_{*} \quad \text { and } \quad \lim _{n \rightarrow \infty} \vartheta_{*}^{-n} \stackrel{\circ}{F}_{*}^{n} \eta=d(\bar{\rho}) \cdot \tilde{\mu}_{*}
$$

where the convergence is in the weak* topology.

(c) $\tilde{\mu}_{*}$ has absolutely continuous conditional measures on unstable leaves.

Remark 4.5. In Sect. 5.1 we show that $\bar{\pi}_{*} \tilde{\mu}_{*}=h_{*} \bar{m}$. Thus $\tilde{\mu}_{*} \in \tilde{\mathcal{G}}$ and the $\vartheta_{*}$ of Theorem 4.2 is the same as the $\vartheta_{*}$ of Theorem 4.4.

Theorem 4.4 treats one hole at a time. The following uniform bounds are also needed, mostly for purposes of proving Theorem 4.

Proposition 4.6. Consider all $(F, \Delta, \tilde{H})$ arising from any $\left(\Lambda^{(\sigma)}, R^{(\sigma)}\right)$ in Proposition 2.2 for which the hole condition in (3) is met. Let $\tilde{\mu}_{*}$ and $\vartheta_{*}$ be as in Theorem 4.4. Then there are constants $C_{2}, K>0$ such that

(i) the conditional densities $\rho_{\gamma}$ of $\left.\tilde{\mu}_{*}\right|_{\Delta_{\ell}}$ with respect to $\mu_{\gamma}$ on unstable leaves satisfy $C_{2}^{-1} \vartheta_{*}^{-\ell} \leq \rho_{\gamma} \leq C_{2} \vartheta_{*}^{-\ell}$

(ii) $\tilde{\mu}_{*}\left(\cup_{\ell>L} \Delta_{\ell}\right) \leq K \beta^{-L} \theta_{0}^{L}$; and

(iii) $\vartheta_{*} \rightarrow 1$ as $n(\Delta, \tilde{H}) \rightarrow \infty$.

\section{Proofs of Theorems on the Tower}

The following notational abbreviations are used only in this section:

- We will sometimes drop the used to distinguish between objects on $M$ and corresponding objects on $\Delta$; there can be no ambiguity as long as we restrict ourselves to the towers.

- We will at times drop the ${ }^{\circ}$ in $\stackrel{\circ}{F}$. Specifically, $F_{*}^{n} \eta$ is to be interpreted as $\stackrel{\circ}{F}_{*}^{n} \eta$, and $\bar{F}_{*}^{n} \bar{\eta}$ is to be interpreted the same way.

We focus on the stable direction, since that is what lies between Theorem 4.2 and Theorem 4.4. The following is a class of test functions on $\stackrel{\triangleright}{\Delta}$ that are Lipschitz in the stable 
direction. For $\gamma^{s} \in \Gamma^{s}(\Delta)$ and $x, y \in \gamma^{s}$, we denote by $d^{s}(x, y)$ the distance between $\pi(x)$ and $\pi(y)$ according to the $p$-metric, so that $d^{s}\left(F^{n} x, F^{n} y\right) \leq \lambda^{-n} d^{s}(x, y)$ for some $\lambda>1$ (see Sect. 2.3). Let $\mathcal{F}_{b}$ be the set of bounded, measurable functions on $\stackrel{\Delta}{~ . ~ F o r ~} \varphi \in \mathcal{F}_{b}$, we define $|\varphi|_{\text {Lip }}^{s}$ to be the Lipshitz constant of $\varphi$ restricted to stable leaves, i.e.

$$
|\varphi|_{\text {Lip }}^{s}=\sup _{\gamma^{s} \in \Gamma^{s}(\stackrel{\Delta}{\Delta})} \sup _{x, y \in \gamma^{s}} \frac{\varphi(x)-\varphi(y)}{d_{s}(x, y)}
$$

and let $\operatorname{Lip}^{s}(\stackrel{ْ}{\Delta})=\left\{\varphi \in \mathcal{F}_{b}:|\varphi|_{\text {Lip }}^{s}<\infty\right\}$

\subsection{Proof of Theorem $\mathbf{4 . 4}$}

\section{A. Escape rates}

Theorem 4.4(a) follows easily from Theorem 4.2 as $(F, \Delta, H)$ and $(\bar{F}, \bar{\Delta}, \bar{H})$ have the same escape rate. In more detail, let $\eta \in \tilde{\mathcal{G}}$ and notice that since $H$ is a union of $\Delta_{\ell, j}$, we have, for each $n, \eta\left(\Delta^{n}\right)=\bar{\eta}\left(\bar{\Delta}^{n}\right)$ where $\bar{\eta}=\bar{\pi}_{*} \eta$. By definition of $\tilde{\mathcal{G}}, \frac{d \bar{\eta}}{d \bar{m}}=\bar{\rho} \in \dot{\mathcal{B}}$ with $d(\bar{\rho})>0$. Theorem 4.2(1) then implies that $\vartheta_{*}^{-n} \overline{\mathcal{L}}^{n} \bar{\rho}$ converges to $d(\bar{\rho}) h_{*}$. Since the convergence is in the $\|\cdot\|$-norm, we may integrate with respect to $\bar{m}$. Noting that $\int_{\bar{\Delta}} \overline{\mathcal{L}}^{n} \bar{\rho} d \bar{m}=\int_{\bar{\Delta}^{n}} \bar{\rho} d \bar{m}=\bar{\eta}\left(\bar{\Delta}^{n}\right)$, we have

$$
\lim _{n \rightarrow \infty} \vartheta_{*}^{-n} \eta\left(\Delta^{n}\right)=\lim _{n \rightarrow \infty} \vartheta_{*}^{-n} \bar{\eta}\left(\bar{\Delta}^{n}\right)=d(\bar{\rho}) .
$$

Thus $-\log \vartheta_{*}$, where $\vartheta_{*}$ is the eigenvalue in Theorem 4.2 , is the common escape rate of $(F, \Delta, H)$ for initial distributions in $\tilde{\mathcal{G}}$.

\section{B. Uniqueness of limiting distributions}

We first prove uniqueness postponing the proof of existence of limiting distributions.

Given $\eta \in \tilde{\mathcal{G}}$, we define a measure $\eta^{s}$ on $\Gamma^{u}(\Delta)$, i.e. a measure transverse to unstable leaves, as follows: Set $\eta^{s}\left(\Gamma^{u}\left(\Delta_{\ell, j}\right)\right)=0$ if $\eta\left(\Delta_{\ell, j}\right)=0$. If $\eta\left(\Delta_{\ell, j}\right) \neq 0$, then $\left.\eta^{s}\right|_{\Gamma^{u}\left(\Delta_{\ell, j}\right)}$ is the factor measure of $\left.\eta\right|_{\Delta_{\ell, j}}$ normalized, and $\left\{\rho d m_{\gamma}, \gamma \in \Gamma^{u}\left(\Delta_{\ell, j}\right)\right\}$ is the disintegration of $\eta$ into measures on unstable leaves. We will use the convention that $\eta^{s}\left(\Delta_{\ell, j}\right)=1$, and $\left.\rho\right|_{\gamma}$ is the density with respect to $m_{\gamma}$, so that $\left.\int \rho\right|_{\gamma} d \eta^{s}(\gamma)=\bar{\rho}$ where $d \bar{\pi}_{*} \eta=\bar{\rho} d \bar{m}$.

Lemma 5.1. Let $\eta_{1}$ and $\eta_{2} \in \tilde{\mathcal{G}}$. Suppose for $i=1,2$, there exists $\mu_{*}^{i}$ such that

$$
\lim _{n \rightarrow \infty} \vartheta_{*}^{-n} F_{*}^{n} \eta_{i}=d\left(\bar{\rho}_{i}\right) \mu_{*}^{i}
$$

where $\bar{\rho}_{i}$ is the density of $\bar{\pi}_{*} \eta_{i}$ Then $\mu_{*}^{1}=\mu_{*}^{2}$.

The crux of the argument for Lemma 5.1 is contained in

Lemma 5.2. Let $\eta_{1}$ and $\eta_{2}$ be as above, and assume $\bar{\rho}_{1}=\bar{\rho}_{2}$. Then for all $\varphi \in \operatorname{Lip}\left({ }^{s}\right)$, $\vartheta_{*}^{-n}\left|F_{*}^{n} \eta_{1}(\varphi)-F_{*}^{n} \eta_{2}(\varphi)\right| \rightarrow 0$ exponentially fast as $n \rightarrow \infty$.

Proof. For $i=1,2$, let $\eta_{i}^{s}$ and $\rho_{i}$ be the (normalized) factor measure and (unnormalized) densities on $\gamma \in \Gamma^{u}(\Delta)$ of $\eta_{i}$ as described above. 
We consider functions which are constant along stable leaves to be defined on both $\stackrel{\Delta}{\Delta}$ and $\bar{\Delta}^{0}$ and do not distinguish between the two versions of such functions. For each $\Delta_{\ell, j}$, let $\hat{\gamma} \in \Gamma^{u}\left(\Delta_{\ell, j}\right)$ be a representative leaf. Then

$$
\left|F_{*}^{n} \eta_{1}(\varphi)-F_{*}^{n} \eta_{2}(\varphi)\right| \leq \sum_{\ell, j} \int_{\hat{\gamma} \cap \Delta_{\ell, j}^{n}} d m_{\hat{\gamma}}\left|\int_{\gamma^{s}} \rho_{1} \varphi \circ F^{n} d \eta_{1}^{s}-\int_{\gamma^{s}} \rho_{2} \varphi \circ F^{n} d \eta_{2}^{s}\right| .
$$

Next fix $x \in \hat{\gamma} \cap \Delta^{n}$ and estimate the integrals on $\gamma^{s}(x)$. Define $\bar{\varphi}_{n}=\int_{\gamma^{s}} \varphi \circ F^{n} d \eta_{1}^{s}$. Then,

$$
\begin{aligned}
\left|\int_{\gamma^{s}} \rho_{1} \varphi \circ F^{n} d \eta_{1}^{s}-\int_{\gamma^{s}} \rho_{2} \varphi \circ F^{n} d \eta_{2}^{s}\right| \leq & \left|\int_{\gamma^{s}} \rho_{1}\left(\varphi \circ F^{n}-\bar{\varphi}_{n}\right) d \eta_{1}^{s}\right|+\left|\int_{\gamma^{s}} \rho_{2}\left(\varphi \circ F^{n}-\bar{\varphi}_{n}\right) d \eta_{2}^{s}\right| \\
& +\left|\int_{\gamma^{s}} \bar{\varphi}_{n} \rho_{1} d \eta_{1}^{s}-\int_{\gamma^{s}} \bar{\varphi}_{n} \rho_{2} d \eta_{2}^{s}\right| .
\end{aligned}
$$

Since $\bar{\varphi}_{n}$ is constant on $\gamma^{s}$ and $\bar{\rho}_{1}=\bar{\rho}_{2}$, the third term above is 0 . For the first two terms, we note that for each $y \in \gamma^{s}(x),\left|\bar{\varphi}_{n}(y)-\varphi \circ F^{n}(y)\right| \leq|\varphi|_{\text {Lip }}^{s} \lambda^{-n}$. Thus

$$
\vartheta_{*}^{-n}\left|F_{*}^{n} \mu_{1}(\varphi)-F_{*}^{n} \mu_{2}(\varphi)\right| \leq \vartheta_{*}^{-n} \sum_{\ell, j} \int_{\bar{\Delta}_{\ell, j}^{n}} 2 \bar{\rho}_{1} d \bar{m}|\varphi|_{\text {Lip }}^{s} \lambda^{-n}=2 \vartheta_{*}^{-n}\left|\overline{\mathcal{L}}^{n} \bar{\rho}_{1}\right|_{1}|\varphi|_{\text {Lip }}^{s} \lambda^{-n},
$$

which proves the lemma since $\vartheta_{*}^{-n}\left|\overline{\mathcal{L}}^{n} \bar{\rho}_{1}\right| \rightarrow d\left(\bar{\rho}_{1}\right)$ by Theorem 4.2 .

Remark 5.3. We have used in the proof above a property of the billiard maps, namely $d^{s}\left(F^{n} x, F^{n} y\right) \leq \lambda^{-n} d^{s}(x, y)$. For general towers, one has only the contraction guaranteed by (P3) which is nonuniform. It is not hard to see that the lemma holds in the more general case with the exponential rate given by $\max \left\{\alpha^{\frac{n}{2}}, \beta^{-n} \theta_{0}^{n}\right\}$ in the place of $\lambda^{-n}$; we leave the proof to the interested reader.

Proof of Lemma 5.1. Let $\bar{\mu}_{*}=h_{*} \bar{m}$ be the conditionally invariant measure given by Theorem 4.2. For $i=1,2$, we have, on the one hand,

$$
\lim _{n \rightarrow \infty} \vartheta_{*}^{-n} \bar{F}_{*}^{n} \bar{\eta}_{i}=d\left(\bar{\rho}_{i}\right) \bar{\mu}_{*},
$$

which follows from Theorem 4.2, and on the other,

$$
\lim _{n \rightarrow \infty} \vartheta_{*}^{-n} \bar{\pi}_{*} F_{*}^{n} \eta_{i}=d\left(\bar{\rho}_{i}\right) \bar{\pi}_{*} \mu_{*}^{i},
$$

which follows from the hypothesis of the lemma. Since $\bar{\pi}_{*} F_{*}^{n} \eta_{i}=\bar{F}_{*}^{n} \bar{\pi}_{*} \eta_{i}$ for each $n \geq 0$, we have $\bar{\pi}_{*} \mu_{*}^{1}=\bar{\mu}_{*}=\bar{\pi}_{*} \mu_{*}^{2}$. Thus $\vartheta_{*}^{-n}\left|F_{*}^{n} \mu_{*}^{1}-F_{*}^{n} \mu_{*}^{2}\right| \rightarrow 0$ as $n \rightarrow \infty$ by Lemma 5.2. But $\vartheta_{*}^{-n} F_{*}^{n} \mu_{*}^{i}=\mu_{*}^{i}$ since $\mu_{*}^{i}$ is conditionally invariant. Hence $\mu_{*}^{1}=\mu_{*}^{2}$.

\section{Convergence to conditionally invariant measure}

For a probability measure $\eta$ on $\stackrel{\Omega}{\Delta},\left|F_{*}^{n} \eta\right|=\eta\left(\Delta^{n}\right)=\bar{\pi}_{*} \eta\left(\bar{\Delta}^{n}\right)$. So for $\eta \in \tilde{\mathcal{G}}$, (4) implies $\lim _{n \rightarrow \infty} \vartheta_{*}^{-n}\left|F_{*}^{n} \eta\right|=d(\bar{\rho})>0$ where $\bar{\rho}$ is the density of $\bar{\eta}=\bar{\pi}_{*} \eta$. More than that is true:

Lemma 5.4. $\vartheta_{*}^{-n} F_{*}^{n} \eta / d(\bar{\rho})$ converges weakly to a conditionally invariant probability measure $\mu_{*}$ as $n \rightarrow \infty$. 
This is half of Theorem 4.4(b). Once we have this, it will follow immediately that

$$
\lim _{n \rightarrow \infty} \frac{F_{*}^{n} \eta}{\left|F_{*}^{n} \eta\right|}=\lim _{n \rightarrow \infty} \frac{\vartheta_{*}^{-n} F_{*}^{n} \eta}{\vartheta_{*}^{-n}\left|F_{*}^{n} \eta\right|}=\mu_{*}
$$

which is the other half.

We will use the following algorithm to "lift" measures from $\bar{\Delta}$ to $\Delta$ : Fix a measure $\mu^{s}$ on $\Gamma^{u}(\Delta)$ with $\mu^{s}\left(\Gamma^{u}\left(\Delta_{\ell, j}\right)\right)=1$. Given $\bar{\eta}$ on $\bar{\Delta}$ with density $\bar{\rho}$, we define $\bar{\pi}_{*}^{-1} \bar{\eta}$ to be the measure on $\Delta$ with the property that restricted to each $\Delta_{\ell, j}, \bar{\pi}_{*}^{-1} \bar{\eta}$ decomposes into the factor measure $\mu^{s}$ and leaf measures $\left\{\rho d m_{\gamma}\right\}$ where $\left.\rho\right|_{\bar{\pi}^{-1}(x)} \equiv \bar{\rho}(x)$. Notice that $\bar{\pi}_{*} \bar{\pi}_{*}^{-1} \bar{\eta}=\bar{\eta}$.

Proof of Lemma 5.4. Our first step is to fix $\varphi \in \operatorname{Lip}^{s}(\stackrel{\circ}{\Delta})$ and show that $\vartheta_{*}^{-n} F_{*}^{n} \eta(\varphi)$ is a Cauchy sequence. For a fixed $\mu^{s}$ as above, and let $\bar{\varphi}_{n}(x)=\int_{\gamma^{s}(x)} \varphi \circ \stackrel{\circ}{F}^{n} d \mu^{s}$. Define $\bar{\eta}=\bar{\pi}_{*} \eta$. Since $\eta \in \tilde{\mathcal{G}}, \bar{\eta}$ has density $\bar{\rho} \in \dot{\mathcal{B}}$ with $d(\bar{\rho})>0$. Then by definition of $\bar{\pi}_{*}^{-1}$,

$$
\left(\bar{\pi}_{*}^{-1} \bar{\pi}_{*} \eta\right)\left(\varphi \circ \stackrel{\circ}{F}^{n}\right)=\sum_{\ell, j} \int_{\Gamma^{u}\left(\Delta_{\ell, j}\right)} d \mu^{s}(\gamma) \int_{\gamma^{u}} \varphi \circ \stackrel{\circ}{F}^{n} \bar{\rho} d m_{\gamma}=\sum_{\ell, j} \int_{\bar{\Delta}_{\ell, j}} \bar{\rho} \bar{\varphi}_{n} d \bar{m}^{\prime} \bar{\pi}_{*} \eta\left(\bar{\varphi}_{n}\right) .
$$

For $n, k_{1}, k_{2} \geq 0$, write

$$
\begin{aligned}
\mid \vartheta_{*}^{-n-k_{1}} F_{*}^{n+k_{1}} \eta(\varphi)-\vartheta_{*}^{-n-k_{2}} & F_{*}^{n+k_{2}} \eta(\varphi)\left|\leq \vartheta_{*}^{-n-k_{1}}\right| F_{*}^{n+k_{1}} \eta(\varphi)-F_{*}^{n} \bar{\pi}_{*}^{-1} \bar{\pi}_{*} F_{*}^{k_{1}} \eta(\varphi) \mid \\
& +\left|\vartheta_{*}^{-n-k_{1}} F_{*}^{n} \bar{\pi}_{*}^{-1} \bar{\pi}_{*} F_{*}^{k_{1}} \eta(\varphi)-\vartheta_{*}^{-n-k_{2}} F_{*}^{n} \bar{\pi}_{*}^{-1} \bar{\pi}_{*} F_{*}^{k_{2}} \eta(\varphi)\right| \\
& +\vartheta_{*}^{-n-k_{2}}\left|F_{*}^{n} \bar{\pi}_{*}^{-1} \bar{\pi}_{*} F_{*}^{k_{2}} \eta(\varphi) \eta(\varphi)-F_{*}^{n+k_{2}} \eta(\varphi)\right| .
\end{aligned}
$$

The first and third terms of (9) are estimated using Lemma 5.2 since $\bar{\pi}_{*}\left(\vartheta_{*}^{-k_{i}} F_{*}^{k_{i}} \eta\right)=$ $\bar{\pi}_{*}\left(\vartheta_{*}^{-k_{i}} \bar{\pi}_{*}^{-1} \bar{\pi}_{*} F_{*}^{k_{i}} \eta\right)$ for $i=1,2$. Thus by Lemma 5.2 ,

$$
\vartheta_{*}^{-n-k_{i}}\left|F_{*}^{n+k_{i}} \eta(\varphi)-F_{*}^{n} \bar{\pi}_{*}^{-1} \bar{\pi}_{*} F_{*}^{k_{i}} \eta(\varphi)\right| \leq C^{\prime} d(\bar{\rho})\left(|\varphi|_{\text {Lip }}^{s}+|\varphi|_{\infty}\right) \zeta^{n}
$$

for some $C^{\prime}>0$ and $\zeta<1$.

We now fix $n$ and estimate the second term of (9). Due to (8), for any $k \geq 0$ we have

$$
\begin{gathered}
\vartheta_{*}^{-n-k} F_{*}^{n} \bar{\pi}_{*}^{-1} \bar{\pi}_{*} F_{*}^{k} \eta(\varphi)=\vartheta_{*}^{-n-k} \bar{\pi}_{*}^{-1} \bar{\pi}_{*} F_{*}^{k} \eta\left(\varphi \circ F^{n} \cdot 1_{\Delta^{n}}\right)=\vartheta_{*}^{-n-k} \bar{\pi}_{*} F_{*}^{k} \eta\left(\bar{\varphi}_{n} \cdot 1_{\bar{\Delta}^{n}}\right) \\
=\vartheta_{*}^{-n-k} \bar{F}_{*}^{k} \bar{\eta}\left(\bar{\varphi}_{n} \cdot 1_{\bar{\Delta}^{n}}\right)=\vartheta_{*}^{-n-k} \int_{\bar{\Delta}^{n}} \bar{\varphi}_{n} \cdot \overline{\mathcal{L}}^{k} \bar{\rho} d \bar{m} .
\end{gathered}
$$

Recalling that $\bar{\rho} \in \dot{\mathcal{B}}$ and $d(\bar{\rho})>0$ since $\eta \in \tilde{\mathcal{G}}$, we estimate

$$
\begin{aligned}
& \left|\vartheta_{*}^{-n-k_{1}} F_{*}^{n} \bar{\pi}_{*}^{-1} \bar{\pi}_{*} F_{*}^{k_{1}} \eta(\varphi)-\vartheta_{*}^{-n-k_{2}} F_{*}^{n} \bar{\pi}_{*}^{-1} \bar{\pi}_{*} F_{*}^{k_{2}} \eta(\varphi)\right| \\
& \leq|\varphi|_{\infty} \vartheta_{*}^{-n} \int_{\bar{\Delta}^{n}}\left|\vartheta_{*}^{-k_{1}} \overline{\mathcal{L}}^{k_{1}} \bar{\rho}-d(\bar{\rho}) h_{*}\right| d \bar{m}+|\varphi|_{\infty} \vartheta_{*}^{-n} \int_{\bar{\Delta}^{n}}\left|\vartheta_{*}^{-k_{2}} \overline{\mathcal{L}}^{k_{2}} \bar{\rho}-d(\bar{\rho}) h_{*}\right| d \bar{m} .
\end{aligned}
$$

Both terms of (10) are small: By Theorem 4.2(1),

$$
\vartheta_{*}^{-n} \int_{\bar{\Delta}^{n}}\left|\vartheta_{*}^{-k} \overline{\mathcal{L}}^{k} \bar{\rho}-d(\bar{\rho}) h_{*}\right| d \bar{m} \leq \vartheta_{*}^{-n}\left\|\vartheta_{*}^{-k} \overline{\mathcal{L}}^{k} \bar{\rho}-d(\bar{\rho}) h_{*}\right\| \int_{\bar{\Delta}^{n}} 1_{\beta} d \bar{m} \leq \vartheta_{*}^{-n}\left|\overline{\mathcal{L}}^{n} 1_{\beta}\right|{ }_{1} D\|\bar{\rho}\| \tau^{k}
$$


for $k=k_{i}$, where $1_{\beta}(x)=\beta^{-\ell}$ for $x \in \stackrel{\circ}{\ell}_{\ell}$. Since $1_{\beta} \in \stackrel{\circ}{\mathcal{B}}, \vartheta_{*}^{-n}\left|\overline{\mathcal{L}}^{n} 1_{\beta}\right|_{1}$ converges to $d\left(1_{\beta}\right)$ as $n \rightarrow \infty$. Thus (10) can be made arbitrarily small by choosing $k_{1}$ and $k_{2}$ sufficiently large.

We have shown that $\vartheta_{*}^{-n} F_{*}^{n} \eta(\varphi) / d(\bar{\rho})$ is a Cauchy sequence and therefore converges to a number $Q(\varphi)$. The functional $Q(\varphi):=\lim _{n \rightarrow \infty} \vartheta_{*}^{-n} F_{*}^{n} \eta(\varphi) / d(\bar{\rho})$ is clearly linear in $\varphi$, positive and satisfies $Q(1)=1$. Also $|Q(\varphi)| \leq|\varphi|_{\infty} Q(1)$ so that $Q$ extends to a bounded

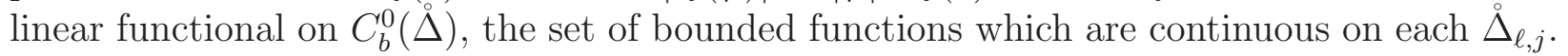

By the Riesz representation theorem, there exists a unique Borel probability measure $\mu_{*}$ satisfying $\mu_{*}(\varphi)=Q(\varphi)$ for each $\varphi \in C_{b}^{0}(\stackrel{\circ}{\Delta})[\mathrm{H}$, Section 56]. Also,

$$
d(\bar{\rho}) \mu_{*}(\varphi \circ \stackrel{\circ}{F})=\lim _{n \rightarrow \infty} \vartheta_{*}^{-n} F_{*}^{n} \eta(\varphi \circ \stackrel{\circ}{F})=\vartheta_{*} \lim _{n \rightarrow \infty} \vartheta_{*}^{-n-1} F_{*}^{n+1} \eta(\varphi)=\vartheta_{*} d(\bar{\rho}) \mu_{*}(\varphi)
$$

so that $\mu_{*}$ is a conditionally invariant measure for $\stackrel{\circ}{F}$ with escape rate $-\log \vartheta_{*}$.

This completes the proof of parts (a) and (b) of Theorem 4.4. To prove part (c), we must show that $\mu_{*}$ has absolutely continuous conditional measures on unstable leaves. Proof of a stronger version of this fact is contained in the proof of Proposition 4.6(i) below.

Notice that from the proof of Lemma 5.1 , we have $\bar{\pi}_{*} \mu_{*}=\bar{\mu}_{*}$ so that the density of $\bar{\pi}_{*} \mu_{*}$ is precisely $h_{*}$. Since $h_{*} \in \dot{\mathcal{B}}$ and $d\left(h_{*}\right)=1>0$, we conclude $\mu_{*} \in \tilde{\mathcal{G}}$.

\subsection{Proof of Proposition 4.6}

Consider the set of holes $\Sigma_{h}\left(q_{0}\right)$ for fixed $q_{0}$ where $h$ is small enough as required in Proposition 2.2. For $\sigma \in \Sigma_{h}\left(q_{0}\right)$, let $\Delta^{(\sigma)}$ be the tower with holes induced by the generalized horseshoe, and let $\mu_{*}^{(\sigma)}$ be the conditionally invariant measure given by Theorem 4.4.

Proof of (i). We drop the superscript $(\sigma)$ in what follows and point out that the constants we use are uniform for all $\sigma \in \Sigma_{h}\left(q_{0}\right)$ and $h$ sufficiently small.

Choose $\gamma_{0} \in \Gamma^{u}\left(\Delta_{0}\right)$ and let $\eta_{0}$ be the measure supported on $\gamma_{0}$ with uniform density with respect to $\mu_{\gamma}$. We claim that $\eta_{0} \in \tilde{\mathcal{G}}$. It is immediate that $\bar{\pi}_{*} \eta_{0}$ has density $\bar{\rho}=\left.e^{-u}\right|_{\gamma_{0}}$ with respect to $\bar{m}$, which is in $\dot{\mathcal{B}}$ by Lemma 4.1 . To see that $d(\bar{\rho})>0$, notice that the mixing assumption on $(\bar{F}, \bar{\Delta})$ implies that $\bar{\Delta}_{0}$ is necessarily a surviving partition element. Since $\bar{\rho}>0$ on $\bar{\Delta}_{0}$, Corollary 4.3 implies that $d(\bar{\rho})>0$.

By Theorem 4.4(b), $\eta^{(n)}:=F_{*}^{n} \eta_{0} /\left|F_{*}^{n} \eta_{0}\right|$ converges to $\mu_{*}$. Let $\rho_{\gamma}^{(n)}$ denote the density of $\eta^{(n)}$ with respect to $\mu_{\gamma}$ on $\gamma \in \Gamma^{u}(\Delta)$. Notice that inverse branches of $\stackrel{\circ}{F}^{n}$ on $\gamma$ are well defined. For any $x_{1}, x_{2} \in \gamma$, treating one branch at a time and summing over all branches, we obtain that

$$
\frac{\rho_{\gamma}^{(n)}\left(x_{1}\right)}{\rho_{\gamma}^{(n)}\left(x_{2}\right)}=\frac{\sum_{y_{1} \in \stackrel{\circ}{F}^{-n} x_{1}}\left(J_{\mu_{\gamma}} \stackrel{\circ}{F}^{n}\left(y_{1}\right)\right)^{-1}}{\sum_{y_{2} \in \stackrel{\circ}{F}^{-n} x_{2}}\left(J_{\mu_{\gamma}} \stackrel{\circ}{F}^{n}\left(y_{2}\right)\right)^{-1}} \leq \sup _{y_{1} \in \stackrel{\circ}{F}^{-n} x_{1}} \frac{J_{\mu_{\gamma}} \stackrel{\circ}{F}^{n}\left(y_{2}\right)}{J_{\mu_{\gamma}} \stackrel{\circ}{F}^{n}\left(y_{1}\right)} \leq e^{C}
$$

by Property $(\mathbf{P} 4)(\mathrm{b})$ where $J_{\mu_{\gamma}} \stackrel{\circ}{F}^{n}$ is the Jacobian of $\stackrel{\circ}{F}^{n}$ with respect to $\mu_{\gamma}$. Since by Proposition 2.2 the constant $C$ is independent of $\sigma, x$ and $n$, we have

$$
e^{-C} \leq \frac{\sup _{x \in \gamma} \rho_{\gamma}^{(n)}(x)}{\inf _{x \in \gamma} \rho_{\gamma}^{(n)}(x)} \leq e^{C}
$$


This estimate plus the minimum length $\kappa$ of $\mu^{u}(\Lambda)$ given by Proposition 2.2 yields the desired uniform upper and lower bounds on the conditional densities of $\eta^{(n)}$ with respect to $\mu_{\gamma}$ (and hence to $m_{\gamma}$ ) on $\Delta_{0}$. The uniformity of these bounds in $n$ implies that they pass to the conditional densities of $\mu_{*}$ in the limit as $n \rightarrow \infty$. Since $\mu_{*}$ is conditionally invariant,

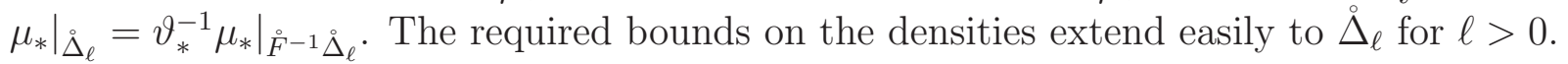

Proof of (ii). We decompose $\mu_{*}$ into a normalized factor measure $\mu_{*}^{s}$ on $\Gamma^{u}\left(\Delta_{\ell}\right)$ and densities $\rho_{\gamma}$ with respect to $m_{\gamma}$ on $\gamma \in \Gamma^{u}\left(\Delta_{\ell}\right)$. Then

$$
\mu_{*}\left(\cup_{\ell \geq L} \Delta_{\ell}^{(\sigma)}\right)=\sum_{\ell \geq L} \int_{\Gamma^{u}\left(\Delta_{\ell}^{(\sigma)}\right)} d \mu_{*}^{s} \int_{\gamma} \rho_{\gamma} d m_{\gamma} \leq \sum_{\ell \geq L} C_{2} \vartheta_{*}^{-\ell} \bar{m}\left(\bar{\Delta}_{\ell}\right) \leq C_{2} \sum_{\ell \geq L} C_{0} \theta_{0}^{\ell} \beta^{-\ell} .
$$

Here we have used Proposition 4.6(i) to estimate $\rho_{\gamma}$, Proposition 2.2 and Lemma 4.1 for the uniformity of $C_{0}$ and $\theta_{0}$, and the fact that $\vartheta_{*}>\beta$. The sum can be made arbitrarily small since $\beta>\theta_{0}$.

Proof of (iii). Notice that $n(\Delta, \tilde{H}) \geq \bar{n}(h)$ by definition of $\bar{n}(h)$ in Sect. 2.2. From Theorem 4.2(2), we know that the escape rate $-\log \vartheta_{*}$ satisfies

$$
\vartheta_{*}>1-\frac{1+C_{1}}{\kappa} \sum_{\ell \geq 1} \beta^{\ell-1} \bar{m}\left(\bar{H} \cap \bar{\Delta}_{\ell}\right)>1-\frac{1+C_{1}}{\kappa} \sum_{\ell \geq \bar{n}(h)} \beta^{\ell-1} C_{0} \theta_{0}^{\ell} .
$$

By Proposition 2.2, $\bar{n}(h) \rightarrow \infty$ as $h \rightarrow 0$, so that $\vartheta_{*} \rightarrow 1$.

\section{Proofs of Theorems for Billiards}

In the Proofs of Theorems 1-3, we fix a hole $\sigma$ that is acceptable with respect to Proposition 2.2 and for which $n\left(\Lambda^{(\sigma)}, R^{(\sigma)}, H_{\sigma}\right)$ is large enough to meet the condition in Theorem 4.4. We suppress mention of $\sigma$, and let $(F, \Delta, \tilde{H})$ be the tower constructed from $(\Lambda, R, H)$. Define $M^{n}=\cap_{i=0}^{n} f^{-n} \stackrel{\circ}{M}, M^{\infty}=\cap_{n \geq 0} M^{n}$.

\subsection{Proof of Theorems 1 and 2}

The first order of business is to show that each $\eta \in \mathcal{G}$ can be lifted to a measure $\tilde{\eta} \in \tilde{\mathcal{G}}$ in such a way that the escape dynamics on $\stackrel{\Delta}{ }$ with initial distribution $\tilde{\eta}$ reflect those on $\dot{M}$ with initial distribution $\eta$. Recall that the natural invariant probability measure for the closed billiard system $f: M \rightarrow M$ is denoted by $\nu$. In [Y, Sect. 2], it is shown that there is a unique invariant probability measure $\tilde{\nu}$ for the tower map $F: \Delta \rightarrow \Delta$ with absolutely continuous conditional measures on unstable leaves, and this measure has the property $\pi_{*} \tilde{\nu}=\nu$. Given $\eta \in \mathcal{G}$, we define $\tilde{\eta}$ on $\Delta$ as follows: By definition, every $\eta \in \mathcal{G}$ is absolutely continuous with respect to $\nu$. Let $\psi=\frac{d \eta}{d \nu}$. We take $\tilde{\eta}$ to be the measure given by $d \tilde{\eta}=\tilde{\psi} d \tilde{\nu}$ where $\tilde{\psi}=\psi \circ \pi$. This implies in particular that $\pi_{*} \tilde{\eta}=\eta$.

Lemma 6.1. If $\eta \in \mathcal{G}$, then $\tilde{\eta} \in \tilde{\mathcal{G}}$. 
As before, let $\mathcal{F}_{b}$ denote the set of bounded functions on $\Delta$. For $\varphi \in \mathcal{F}_{b}$ and $\gamma \in \Gamma^{u}(\Delta)$, we let $\operatorname{Lip}^{u}\left(\left.\varphi\right|_{\gamma}\right)$ be the Lipschitz constant of $\left.\varphi\right|_{\gamma}$ with respect to the $d_{\beta}$-metric (notice that $d_{\beta}$, the symbolic metric defined on $\bar{\Delta}$, can be thought of as a metric on unstable leaves). Let

$$
|\varphi|_{\text {Lip }}^{u}=\sup _{\gamma \in \Gamma^{u}(\Delta)} \operatorname{Lip}^{u}\left(\left.\varphi\right|_{\gamma}\right)
$$

and $\operatorname{Lip}^{u}(\Delta)=\left\{\varphi \in \mathcal{F}_{b}:|\varphi|_{\text {Lip }}^{u}<\infty\right\}$. The first step toward proving Lemma 6.1 is

Lemma 6.2. Let $\varphi: M \rightarrow \mathbb{R}$ be Lipschitz. Then $\tilde{\varphi}:=\varphi \circ \pi \in \operatorname{Lip}^{u}(\Delta)$ with $|\tilde{\varphi}|_{\text {Lip }}^{u} \leq C \operatorname{Lip}(\varphi)$.

Proof. Recall that for $x, y \in M$ lying in a piece of local unstable manifold, we have $d(x, y) \leq$ $p(x, y)^{1 / 2}$ where $p(\cdot, \cdot)$ is the $p$-metric (see Sect. 2.3). Now for $\gamma \in \Gamma^{u}(\Delta)$ and $x, y \in \gamma$, we have

$$
|\tilde{\varphi}(x)-\tilde{\varphi}(y)|=|\varphi(\pi x)-\varphi(\pi y)| \leq \operatorname{Lip}(\varphi) d(\pi x, \pi y) \leq \operatorname{Lip}(\varphi) p(\pi x, \pi y)^{\frac{1}{2}} .
$$

By (P4)(a), $p(\pi x, \pi y)^{\frac{1}{2}} \leq C \alpha^{s(\pi x, \pi y) / 2}$, which is $\leq C d_{\beta}(x, y)$ since $s \leq s_{0}$ and $\beta \geq \sqrt{\alpha}$.

Proof of Lemma 6.1. (i) First we show $\bar{\pi}_{*} \tilde{\eta}=\bar{\rho} \bar{m}$ with $\bar{\rho} \in \dot{\mathcal{B}}$. Let $\psi=\frac{d \eta}{d \nu}$. Then disintegrating $\tilde{\eta}$ into $\tilde{\eta}^{s}$ and $\left\{\rho_{\gamma} d m_{\gamma}, \gamma \in \Gamma^{u}(\Delta)\right\}$, we obtain

$$
\rho_{\gamma}:=\tilde{\psi} \cdot \frac{d \tilde{\nu}}{d \mu_{\gamma}} \cdot \frac{d \mu_{\gamma}}{d m_{\gamma}} .
$$

Now $\tilde{\psi}$ is bounded by assumption and is $\in \operatorname{Lip}^{u}(\Delta)$ by Lemma 6.2 , $\frac{d \tilde{\nu}}{d \mu_{\gamma}}$ is bounded and is $\in \operatorname{Lip}^{u}(\Delta)\left([Y]\right.$, Sect. 2), as is $\frac{d \mu_{\gamma}}{d m_{\gamma}}$ (Lemma 4.1). Thus we conclude that $\rho_{\gamma} \in \operatorname{Lip}^{u}(\Delta)$ and is bounded. Recall that $\bar{\rho}(x)=\int_{\gamma^{s}(x)} \rho_{\gamma} d \tilde{\eta}^{s}$. It follows immediately that $|\bar{\rho}|_{\infty} \leq \sup _{\gamma}\left|\rho_{\gamma}\right|_{\infty}$ and $\operatorname{Lip}(\bar{\rho}) \leq \sup _{\gamma} \operatorname{Lip}^{u}\left(\rho_{\gamma}\right)$.

(ii) It remains to show $d(\bar{\rho})>0$. By definition of $\mathcal{G}, \psi>0$ on $M^{\infty}$, the set of points which never escape from $M$, so $\tilde{\psi}>0$ on $\Delta^{\infty}$. The fact that $d \tilde{\nu} / d \mu_{\gamma}$ and $e^{-u}$ are strictly positive implies that $\bar{\rho}>0$ on $\Delta^{\infty}$; hence it is $>0$ on a surviving cylinder set, i.e. a set $E_{k}$ such that $\bar{F}^{k}$ maps $E_{k}$ onto a surviving $\bar{\Delta}_{\ell, j}$ before any part of it enters the hole. By Corollary 4.3, $d\left(\overline{\mathcal{L}}^{k} \bar{\rho}\right)>0$. Since $\int_{\bar{\Delta}^{n}} g d \bar{m}=\int_{\bar{\Delta}} \overline{\mathcal{L}}^{n} g d \bar{m}$ for each $n \geq 0$ and $g \in L^{1}(\bar{m})$, we have $d\left(\overline{\mathcal{L}}^{k} \bar{\rho}\right)=\vartheta_{*}^{k} d(\bar{\rho})$ so that $d(\bar{\rho})>0$ as well.

Proof of Theorems 1 and 2 . Given $\eta \in \mathcal{G}$, let $\tilde{\eta}$ be as defined earlier. Then $\tilde{\eta} \in \tilde{\mathcal{G}}$ by Lemma 6.1. For $\varphi \in C^{0}(M)$, let $\tilde{\varphi}=\varphi \circ \pi$. Then $\tilde{\varphi} \in C_{b}^{0}(\Delta)$ and for $n \geq 0$ we have,

$$
\stackrel{\circ}{f}_{*}^{n} \eta(\varphi)=\eta\left(\varphi \circ f^{n} \cdot 1_{M^{n}}\right)=\tilde{\eta}\left(\tilde{\varphi} \circ F^{n} \cdot 1_{\Delta^{n}}\right)=\stackrel{\circ}{*}_{*}^{n} \tilde{\eta}(\tilde{\varphi}) .
$$

Setting $\varphi \equiv 1$ in $(12)$, we have $\eta\left(M^{n}\right)=\stackrel{\circ}{f}_{*}^{n} \eta(\stackrel{\circ}{M})=\stackrel{\circ}{F}_{*}^{n} \tilde{\eta}(\stackrel{\circ}{\Delta})=\tilde{\eta}\left(\Delta^{n}\right)$ for $n>0$, so

$$
\lim _{n \rightarrow \infty} \frac{1}{n} \log \eta\left(M^{n}\right)=\lim _{n \rightarrow \infty} \frac{1}{n} \log \tilde{\eta}\left(\Delta^{n}\right)=\log \vartheta_{*}
$$

by Theorem 4.4(a). This proves Theorem 1. 
Let $\mu_{*}=\pi_{*} \tilde{\mu}_{*}$ where $\tilde{\mu}_{*}$ is given by Theorem 4.4. Then $\mu_{*}(\varphi)=\tilde{\mu}_{*}(\tilde{\varphi})$, and

$$
\stackrel{\circ}{f}_{*} \mu_{*}(\varphi)=\stackrel{\circ}{F}_{*} \tilde{\mu}_{*}(\tilde{\varphi})=\vartheta_{*} \tilde{\mu}_{*}(\tilde{\varphi})=\vartheta_{*} \mu_{*}(\varphi),
$$

proving $\mu_{*}$ is conditionally invariant. Using (12) again, the fact that the normalizations are equal, and Theorem 4.4(b), we obtain

$$
\lim _{n \rightarrow \infty} \frac{\stackrel{\circ}{f}_{*}^{n} \eta(\varphi)}{f_{*}^{n} \eta(M)}=\lim _{n \rightarrow \infty} \frac{\stackrel{\circ}{F}_{*}^{n} \tilde{\eta}(\tilde{\varphi})}{\dot{\circ}_{*}^{n} \eta(\stackrel{\circ}{\Delta})}=\tilde{\mu}_{*}(\tilde{\varphi})=\mu_{*}(\varphi) .
$$

Thus $f_{*}^{n} \eta / \eta\left(M^{n}\right) \rightarrow \mu_{*}$ weakly. Finally,

$$
\lim _{n \rightarrow \infty} \vartheta_{*}^{-n} \stackrel{\circ}{*}_{*}^{n} \eta(\varphi)=\lim _{n \rightarrow \infty} \vartheta_{*}^{-n} \stackrel{\circ}{F}_{*}^{n} \tilde{\eta}(\tilde{\varphi})=d(\bar{\rho}) \cdot \tilde{\mu}_{*}(\tilde{\varphi})=d(\bar{\rho}) \cdot \mu_{*}(\varphi),
$$

where $d(\bar{\rho})>0$ since $\tilde{\eta} \in \tilde{\mathcal{G}}$. This completes the proof of Theorem 2 .

Remark 6.3. In the proof of Lemma 6.1, step (i) holds for any $\eta$ that has Lipschitz densities on unstable leaves. Thus for this class of measures, Theorem 2(b) holds (with $c(\eta)$ possibly equal to zero). It is also clear from step (ii) that to show $d(\bar{\rho})>0$, it suffices to assume $\psi>0$ on $M^{\infty} \cap \Lambda$, or on $M^{\infty} \cap \pi\left(\Delta_{\ell, j}\right)$ where $\bar{\pi}\left(\Delta_{\ell, j}\right)$ is any surviving element.

\subsection{Proof of Theorem 3}

Let $\mu_{*}=\pi_{*} \tilde{\mu}_{*}$ be as above.

(a) Since $\stackrel{\circ}{*}_{*} \mu_{*}=\vartheta_{*} \mu_{*}$, it follows that $\mu_{*}$ is supported on $M \backslash \cup_{n \geq 0} f^{n}(H)$ where $H=H_{\sigma}$. This set has Lebesgue measure zero since by the ergodicity of $f, \cup_{n \geq 0} f^{n}(H)$ has full Lebesgue measure. Thus $\mu_{*}$ is singular with respect to Lebesgue measure.

(b) First, we argue that $\mu_{*}$ has absolutely continuous conditional measures on unstable leaves (without claiming that the densities are strictly positive). This is true because for each $\ell, j$, $\left.\tilde{\mu}_{*}\right|_{\Delta_{\ell, j}}$ has absolutely continuous conditional measures on $\gamma \in \Gamma^{u}\left(\Delta_{\ell, j}\right)$, and $\left.\pi\right|_{\Delta_{\ell, j}}$, which is one-to-one, identifies each $\gamma$ with a positive Lebesgue measure subset of a local unstable manifold of $f$.

The rest of the proof is concerned with showing that the conditional densities of $\mu_{*}$ are strictly positive. To do that, it is not productive to view $\mu_{*}$ as $\pi_{*} \tilde{\mu}_{*}$. Instead, we will view $\mu_{*}$ as the weak limit of $\nu^{(n)}:=f_{*}^{n} \nu /\left|f_{*}^{n} \nu\right|$ as $n \rightarrow \infty$ where $\nu$ is the natural invariant measure for $f$. This convergence of $\nu^{(n)}$ is guaranteed by Theorem 2 . We will prove that $\mu_{*}$ has the properties immediately following the statement of Theorem 3 in Sect. 1.3.

Step 1: Our first patch is built on $V=\cup\left\{\gamma^{u}: \gamma^{u} \in \Gamma^{u}(\Lambda)\right\}$ where $\Gamma^{u}=\Gamma^{u}(\Lambda)$ is the defining family of unstable curves for $\Lambda$. To understand the geometric properties of $\left.\nu^{(n)}\right|_{V}$, observe that in backward time, each $\gamma^{u} \in \Gamma^{u}$ either falls into the hole completely or stays out completely. This is because $f(\partial H)$ is regarded as part of the discontinuity set for $f^{-1}$ when we constructed the horseshoe $\Lambda$ (see Sect. 3.2). Thus there is a decreasing sequence of sets $U_{n}=\cup\left\{\gamma^{u} \in \Gamma^{u}: f^{-i} \gamma^{u} \cap H=\emptyset\right.$ for all $\left.0 \leq i \leq n\right\} \subset V$ consisting of whole $\gamma^{u}$-curves. Assuming $\nu\left(U_{n}\right)>0$ for now, we have $\left.\nu^{(n)}\right|_{V}=\left.c_{n} \nu\right|_{U_{n}}$ for some constant $c_{n}>0$ as $\nu$ is $f$-invariant. Let $\zeta$ be a limit point of $\left.\nu^{(n)}\right|_{V}$, i.e., $\zeta=\left.\lim _{n_{k}} \nu^{\left(n_{k}\right)}\right|_{V}$. Assuming $\zeta(V)>0$, 
lower bounds for conditional probability densities of $\left.\nu^{\left(n_{k}\right)}\right|_{V}$, equivalently those of $\left.\nu\right|_{U_{n}}$, are passed to $\zeta$, and these bounds are strictly positive.

To see that $\zeta(V)>0$, recall that $\nu=\pi_{*} \tilde{\nu}$ for some $\tilde{\nu}$ on the tower $\Delta$, so that $\nu^{(n)}=\pi_{*} \tilde{\nu}^{(n)}$ where $\tilde{\nu}^{(n)}=\stackrel{\circ}{F}_{*}^{n} \tilde{\nu} /\left|\stackrel{\circ}{F}_{*}^{n} \tilde{\nu}\right|$. Since $\pi\left(\Delta_{0}\right) \subset V$, we have

$$
\zeta=\left.\lim _{n_{k}} \nu^{\left(n_{k}\right)}\right|_{V} \geq \lim _{n_{k}} \pi_{*}\left(\left.\tilde{\nu}^{\left(n_{k}\right)}\right|_{\Delta_{0}}\right)=\pi_{*}\left(\left.\tilde{\mu}_{*}\right|_{\Delta_{0}}\right) .
$$

We have written an inequality (as opposed to equality) above because parts of $\Delta_{\ell}$ for $\ell \geq 1$ may get mapped into $V$ as well. Clearly, $\tilde{\mu}_{*}\left(\Delta_{0}\right)>0$, thereby ensuring $\zeta(V)>0$, hence $\nu\left(U_{n}\right)>0$ and the strictly positive conditional densities property above. This together with $\zeta \leq\left.\mu_{*}\right|_{V}$ (equality is not claimed because it is possible for part of $\nu^{\left(n_{k}\right)}$ from outside of $V$ to leak into $V$ in the limit) proves that $(V, \zeta)$ is an acceptable patch.

Step 2: Next we use $(V, \zeta)$ to build patches $\left(V_{\ell, j}, \zeta_{\ell, j}\right)$ corresponding to partition elements $\Delta_{\ell, j}$ of the tower $\Delta$ with $\ell>0$ and $\tilde{\mu}_{*}\left(\Delta_{\ell, j}\right)>0$. From Sections 3 and 4 , we know that $\pi\left(\Delta_{\ell, j}\right)$ is a hyperbolic product set, and $\pi\left(\Delta_{\ell, j}\right)=f^{\ell}\left(\Lambda^{s}\right)$ for some $s$-subset $\Lambda^{s} \subset \Lambda$. Moreover, $f^{i}\left(\Lambda^{s}\right) \cap H=\emptyset$ for all $0<i \leq \ell$. Thus we may assume $V_{\ell, j}=\cup\left\{\gamma^{u}: \gamma^{u} \in \Gamma^{u}\left(\pi\left(\Delta_{\ell, j}\right)\right)\right\} \subset$ $f^{\ell}(V)$. Let $\zeta_{\ell, j}=\left.\vartheta_{*}^{-\ell}\left(f_{*}^{\ell} \zeta\right)\right|_{V_{\ell, j}}$. Then $\zeta_{\ell, j}$ has strictly positive conditional densities on unstable curves because $\zeta$ does, and $\zeta_{\ell, j} \leq\left.\mu_{*}\right|_{V_{\ell, j}}$ as $\mu_{*}$ satisfies $\AA_{*} \mu_{*}=\vartheta_{*} \mu_{*}$.

Finally, since $\zeta_{\ell, j} \geq \pi_{*}\left(\left.\tilde{\mu}_{*}\right|_{\Delta_{\ell, j}}\right)$ for each $\ell, j$, it follows that $\sum_{\ell, j} \zeta_{\ell, j} \geq \mu_{*}$, completing the proof of Theorem 3.

\subsection{Proof of Theorem 4}

Suppose $h_{n}$ is a sequence of numbers tending to $0, \sigma_{h_{n}} \in \Sigma_{h_{n}}\left(q_{0}\right)$ is a sequence of holes in the billiard table, and $H_{n}=H_{\sigma_{n}}$ the corresponding holes in $M$. For each $n$, let $\vartheta_{n}$ be the escape rate and $\mu_{n}$ the physical measure for the leaky system $\left(f, M, H_{n}\right)$ given by Theorem 2 . By Proposition 4.6(iii), we have $\vartheta_{n} \rightarrow 1$ as $n \rightarrow \infty$. To prove $\mu_{n} \rightarrow \nu$, we will assume, having passed to a subsequence, that $\mu_{n}$ converges weakly to some $\mu_{\infty}$, and show that (i) $\mu_{\infty}$ is $f$-invariant, and (ii) it has absolutely continuous conditional measures on unstable leaves. These two properties together uniquely characterize $\nu$.

The following notation will be used: $\Lambda(n)$ is the generalized horseshoe respecting the hole $H_{n}, \Delta(n)=\cup_{\ell} \Delta_{\ell}(n)$ is the corresponding tower, $F_{n}: \Delta(n) \rightarrow \Delta_{n}$ is the tower map, $\pi_{n}: \Delta(n) \rightarrow M$ is the projection, and $\tilde{\mu}_{n}$ is the conditionally invariant measure on $\Delta(n)$ that projects to $\mu_{n}$.

(i) Proof of $f$-invariance: Let $S=\partial M \cup f^{-1} \partial M$.

Lemma 6.4. $\mu_{\infty}(S)=0$

Proof. Let $\delta_{1}$ and $\lambda_{1}$ be as in Sect. 2.3, and let $N_{\varepsilon}(S)$ denote the $\varepsilon$-neighborhood of $S$. We claim that there exist constants $C_{3}, \varsigma>0$ such that for $\varepsilon<\delta_{1}$ and for all $n, \mu_{n}\left(N_{\varepsilon}(S)\right) \leq C_{3} \varepsilon^{\varsigma}$. By the construction of $\Lambda=\Lambda(n)$, any $n, d\left(f^{\ell}(\Lambda), S\right) \geq \delta_{1} \lambda_{1}^{-\ell}$. Thus $f^{\ell}(\Lambda) \cap N_{\varepsilon}(S)=\emptyset$ for all $\ell \leq-\log \left(\varepsilon / \delta_{1}\right) / \log \lambda_{1}$. Hence

$$
\mu_{n}\left(N_{\varepsilon}(S)\right) \leq \sum_{\ell>-\log \left(\varepsilon / \delta_{1}\right) / \log \lambda_{1}} \tilde{\mu}_{n}\left(\Delta_{\ell}(n)\right),
$$


which by Proposition 4.6(ii) is $\leq K\left(\beta^{-1} \theta_{0}\right)^{-\log \left(\varepsilon / \delta_{1}\right) / \log \lambda_{1}}$, proving the claim above with $C_{3}=K / \delta_{1}$ and $\varsigma=\log \left(\beta \theta_{0}^{-1}\right) / \log \lambda_{1}$. Since $C_{3}$ and $\varsigma$ are independent of $n$, these bounds pass to $\mu_{\infty}$, implying $\mu_{\infty}(S)=0$.

Having established that $f$ is well defined $\mu_{\infty}$-a.e., we now verify that $\mu_{\infty}$ is $f$-invariant: Let $\varphi: M \rightarrow \mathbb{R}$ be a continuous function. Then

$$
\int(\varphi \circ f) d \mu_{\infty}=\lim _{n \rightarrow \infty} \int(\varphi \circ f) d \mu_{n}=\lim _{n \rightarrow \infty} \int \varphi d\left(f_{*} \mu_{n}\right)
$$

and

$$
\int \varphi d\left(f_{*} \mu_{n}\right)=\int_{M \backslash H_{n}} \varphi d\left(f_{*} \mu_{n}\right)+\int_{H_{n}} \varphi d\left(f_{*} \mu_{n}\right) .
$$

Since $\left.\left(f_{*} \mu_{n}\right)\right|_{M \backslash H_{n}}=\stackrel{\circ}{*}_{*} \mu_{n}=\vartheta_{n} \mu_{n}$, the first integral on the right side of (13) is equal to $\vartheta_{n} \int \varphi d \mu_{n}$, while the absolute value of the second is bounded by $\left.\left.\left(1-\vartheta_{n}\right)\right|_{\varphi}\right|_{\infty}$. Since $\vartheta_{n} \rightarrow 1$ as $n \rightarrow \infty$, the right side of (13) tends to $\int \varphi d \mu_{\infty}$.

(ii) Absolutely continuous conditional measures on unstable leaves: Since the measures $\tilde{\mu}_{n}$ do not live on the same space for different $n$, a first task here is to find common domains in $M$ on which $\left(\pi_{n}\right)_{*} \tilde{\mu}_{n}$ can be compared. In the constructions to follow, the discontinuity set refers to the real discontinuity set of $f$, not the ones that include boundaries of holes (as was done in Sect. 3).

We choose a rectangular region $\check{Q}$ slightly larger than $Q$ in Proposition 2.2, large enough that $\check{Q} \supset \Lambda(n)$ for all $n$, and let $\check{\Gamma}^{u}$ denote the set of all homogeneous unstable curves connecting the two components of $\partial^{s} \check{Q}$. Let $\check{V}=\cup\left\{\gamma^{u} \in \check{\Gamma}^{u}\right\}$. Then $\Lambda(n) \subset \check{V}$ for all $n$, for $\gamma \cap \check{Q} \in \check{\Gamma}^{u}$ for every $\gamma \in \Gamma^{u}(\Lambda(n))$ (defined using the enlarged discontinuity set). Now for all $n,\left(\pi_{n}\right)_{*}\left(\left.\tilde{\mu}_{n}\right|_{\Delta_{0}(n)}\right)$ is a sequence of measures on $\check{V}$ with absolutely continuous conditional measures on the elements of $\check{\Gamma}^{u}$. Moreover, the conditional densities are uniformly bounded from above with a bound independent of $n$ (Proposition 4.6(i)). Let $\mu_{\infty, 0}$ be a limit point of $\left(\pi_{n}\right)_{*}\left(\left.\tilde{\mu}_{n}\right|_{\Delta_{0}(n)}\right)$. Assuming $\mu_{\infty, 0}(\check{V})>0$, these density bounds are inherited by $\mu_{\infty, 0}$. To show $\mu_{\infty, 0}(\tilde{V})>0$, we will argue there exists $b>0$ such that $\tilde{\mu}_{n}\left(\Delta_{0}(n)\right)>b$ for all $n$, and that is true because the $\tilde{\mu}_{n}$ are probability measures, there is a uniform lower bound on $\tilde{\mu}_{n}\left(\cup_{\ell<L} \Delta_{\ell}(n)\right)$ for large enough $L$ (Proposition 4.6(ii)), and $\tilde{\mu}_{n}\left(\Delta_{\ell+1}(n)\right) \leq \vartheta_{n}^{-1} \tilde{\mu}_{n}\left(\Delta_{\ell}(n)\right)$.

For $\ell>0$, we define $\check{Q}_{\ell}$ to be the finite union of $s$-subrectangles of $\mathscr{Q}$ retained in $\ell$ steps in the construction of $\Lambda$ when $f$ has no holes, i.e. roughly speaking, $\mathscr{Q}_{\ell}$ consists of points that stay away from $S=\partial M \cup f^{-1} \partial M$ by a distance $\geq \delta_{1} \lambda_{1}^{-i}$ at step $i$. Let $\check{V}_{\ell}=\check{V} \cap \check{Q}_{\ell}$. Then $\pi_{n}\left(\stackrel{\circ}{F}_{n}^{-\ell} \Delta_{\ell}(n)\right) \subset \check{V}_{\ell}$ for all $n$. In fact, for each $j, \pi_{n}\left(\stackrel{\circ}{F}_{n}^{-\ell} \Delta_{\ell, j}(n)\right)$ is contained in a connected component of $\check{Q}_{\ell}$. The argument for $\mu_{\infty, 0}$ can now be repeated to conclude the existence of a limit point of $\left(\pi_{n} \circ \stackrel{\circ}{F}_{n}^{-\ell}\right)_{*}\left(\left.\tilde{\mu}_{n}\right|_{\Delta_{\ell}(n)}\right)$ with absolutely continuous conditional measures on unstable leaves. Pushing all measures forward by $f_{*}^{\ell}$, this gives a limit point $\mu_{\infty, \ell}$ of $\left(\pi_{n}\right)_{*}\left(\left.\tilde{\mu}_{n}\right|_{\Delta_{\ell}(n)}\right)$ as $n \rightarrow \infty$ with the same property.

To proceed systematically, we perform a Cantor diagonal argument, choosing a single subsequence $n_{k}$ with the property that for each $\ell \geq 0,\left(\pi_{n_{k}}\right)_{*}\left(\left.\tilde{\mu}_{n_{k}}\right|_{\Delta_{\ell}\left(n_{k}\right)}\right)$ converges to a measure $\mu_{\infty, \ell}$ on $f^{\ell} \check{V}_{\ell}$. Finally, to conclude $\mu_{\infty}=\sum_{\ell} \mu_{\infty, \ell}$, we need a tightness condition as the towers are noncompact. This is given by Proposition 4.6(ii).

The proof of Theorem 4 is now complete. 


\section{References}

[BaK] V. Baladi and G. Keller, Zeta functions and transfer operators for piecewise monotonic transformations, Comm. Math. Phys. 127 (1990), 459-477.

[BDM] H. Bruin, M. Demers, I. Melbourne, Convergence properties and an equilibrium principle for certain dynamical systems with holes, to appear in Ergod. Th. and Dynam. Sys.

[BSC1] L.A. Bunimovich, Ya. G. Sină, and N. I. Chernov, Markov partitions for two-dimensional hyperbolic billiards, Russian Math. Surveys, 45:3 (1990), 105-152.

[BSC2] L.A. Bunimovich, Ya. G. Sină, and N. I. Chernov, Statistical properties of twodimensional hyperbolic billiards, Russian Math. Surveys, 46:4 (1991).

[B] J. Buzzi, Markov extensions for multidimensional dynamical systems, Israel J. of Math. 112 (1999), 357-380.

[C1] N.N. Cenvoca, A natural invariant measure on Smale's horseshoe, Soviet Math. Dokl. 23 (1981), 87-91.

[C2] N.N. Cenvoca, Statistical properties of smooth Smale horseshoes, in Mathematical Problems of Statistical Mechanics and Dynamics, R.L. Dobrushin, ed. Reidel: Dordrecht, 1986, pp. 199-256.

[CM1] N. Chernov and R. Markarian, Ergodic properties of Anosov maps with rectangular holes, Bol. Soc. Bras. Mat. 28 (1997), 271-314.

[CM2] N. Chernov and R. Markarian, Anosov maps with rectangular holes. Nonergodic cases, Bol. Soc. Bras. Mat. 28 (1997), 315-342.

[CM3] N. Chernov and R. Markarian, Chaotic Billiards, Number 127 in Mathematical Surveys and Monographs, American Mathematical Society, 2006.

[CMT1] N. Chernov, R. Markarian and S. Troubetzkoy, Conditionally invariant measures for Anosov maps with small holes, Ergod. Th. and Dynam. Sys. 18 (1998), 1049-1073.

[CMT2] N. Chernov, R. Markarian and S. Troubetzkoy, Invariant measures for Anosov maps with small holes, Ergod. Th. and Dynam. Sys. 20 (2000), 1007-1044.

[CMM] P. Collet, S. Martínez and V. Maume-Deschamps, On the existence of conditionally invariant probability measures in dynamical systems, Nonlinearity 13 (2000), 1263-1274.

[CMS1] P. Collet, S. Martínez and B. Schmitt, The Yorke-Pianigiani measure and the asymptotic law on the limit Cantor set of expanding systems, Nonlinearity 7 (1994), 1437-1443.

[CMS2] P. Collet, S. Martínez and B. Schmitt, Quasi-stationary distribution and Gibbs measure of expanding systems, in Instabilities and Nonequilibrium Structures V, E. Tirapegui and W. Zeller, eds. Kluwer: Dordrecht, 1996, pp. 205-219.

[CMS3] P. Collet, S. Martínez and B. Schmitt, The Pianigiani-Yorke measure for topological Markov chains, Israel J. Math. 97 (1997), 61-70.

[CV] N. Chernov and H. van dem Bedem, Expanding maps of an interval with holes, Ergod. Th. and Dynam. Sys. 22 (2002), 637-654. 
[D1] M. Demers, Markov Extensions for Dynamical Systems with Holes: An Application to Expanding Maps of the Interval, Israel J. of Math. 146 (2005), 189-221.

[D2] M. Demers, Markov Extensions and Conditionally Invariant Measures for Certain Logistic Maps with Small Holes, Ergod. Th. and Dynam. Sys. 25:4 (2005), 1139-1171.

[DL] M. Demers and C. Liverani, Stability of statistical properties in two-dimensional piecewise hyperbolic maps, Trans. Amer. Math. Soc. 360:9 (2008), 4777-4814.

[DY] M. Demers and L.-S. Young, Escape rates and conditionally invariant measures, Nonlinearity 19 (2006), 377-397.

[FKMP] P.A. Ferrari, H. Kesten, S. Martínez and P. Picco, Existence of quasi-stationary distributions. A renewal dynamical approach, Annals of Prob. bf 23:2 (1995), 501-521.

[H] P.R. Halmos, Measure Theory, University Series in Higher Mathematics, D. Van Nostrand Co., Inc.: Princeton, 1950, 304 p.

[HY] A. Homburg and T. Young, Intermittency in families of unimodal maps, Ergod. Th. and Dynam. Sys. 22:1 (2002), 203-225.

[KS] A. Katok and J. M. Strelcyn, Invariant Manifolds, Entropy and Billiards; Smooth Maps with Singularities, volume 1222, Springer Lecture Notes in Math., 1986.

[LiM] C. Liverani and V. Maume-Deschamps, Lasota-Yorke maps with holes: conditionally invariant probability measures and invariant probability measures on the survivor set, Annales de l'Institut Henri Poincaré Probability and Statistics, 39 (2003), 385-412.

[LM] A. Lopes and R. Markarian, Open Billiards: Cantor sets, invariant and conditionally invariant probabilities, SIAM J. Appl. Math. 56 (1996), 651-680.

[PY] G. Pianigiani and J. Yorke, Expanding maps on sets which are almost invariant: decay and chaos, Trans. Amer. Math. Soc. 252 (1979), 351-366.

[R] P.A. Richardson, Jr., Natural measures on the unstable and invariant manifolds of open billiard dynamical systems, Doctoral Dissertation, Department of Mathematics, University of North Texas, 1999.

[S] Ya. G. Sină̌, Dynamical systems with elastic collisions. Ergodic properties of dispersing billiards, Uspehi Mat. Nauk, 25:2 (1970), 141-192.

[V] D. Vere-Jones, Geometric ergodicity in denumerable Markov chains, Quart. J. Math. 13 (1962), 7-28.

[W] Maciej Wojtkowski, Invariant families of cones and Lyapunov exponents, Ergodic Theory Dynam. Systems, 5:1 (1985), 145-161.

[Y] L.-S. Young, Statistical properties of dynamical systems with some hyperbolicity, Annals of Math. 147:3 (1998), 585-650. 